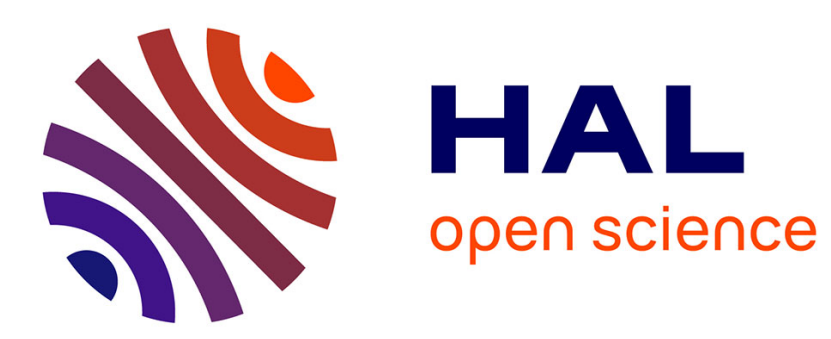

\title{
Computing representations for radicals of finitely generated differential ideals
}

François Boulier, Daniel Lazard, François Ollivier, Michel Petitot

\section{To cite this version:}

François Boulier, Daniel Lazard, François Ollivier, Michel Petitot. Computing representations for radicals of finitely generated differential ideals. Applicable Algebra in Engineering, Communication and Computing, 2009, 20 (1), pp.73-121. 10.1007/s00200-009-0091-7 . hal-00820902

\section{HAL Id: hal-00820902 https://hal.science/hal-00820902}

Submitted on 6 May 2013

HAL is a multi-disciplinary open access archive for the deposit and dissemination of scientific research documents, whether they are published or not. The documents may come from teaching and research institutions in France or abroad, or from public or private research centers.
L'archive ouverte pluridisciplinaire HAL, est destinée au dépôt et à la diffusion de documents scientifiques de niveau recherche, publiés ou non, émanant des établissements d'enseignement et de recherche français ou étrangers, des laboratoires publics ou privés. 
This is the author's version of the article: F. Boulier, D. Lazard, F. Ollivier and M. Petitot, "Computing representations for radicals of finitely generated differential ideals", Special issue "Jacobi's Legacy" of $A A E C C, \mathbf{2 0},(1), 73-$ 121, 2009.

DOI $10.1007 / \mathrm{s} 00200-009-0091-7$
See also F. Boulier, "Foreword to the article: Computing representations for radicals of finitely generated differential ideals", Special issue "Jacobi's Legacy" of $A A E C C, \mathbf{2 0}$, (1), 5-6, 2009.

DOI 10.1007/s00200-009-0089-1

\title{
Computing representations for radicals of finitely generated differential ideals
}

\author{
François Boulier* Daniel Lazard François Ollivier \\ Michel Petitot
}

Résumé

\begin{abstract}
Ce papier s'intéresse aux systèmes d'équations différentielles polynomiales, ordinaires ou aux dérivées partielles. La théorie sousjacente est l'algèbre différentielle de Ritt et Kolchin. Nous décrivons un algorithme, nommé Rosenfeld-Gröbner, qui calcule une représentation du radical $\mathfrak{p}$ de l'idéal différentiel engendré par n'importe quel système $\Sigma$ de cette nature. La représentation calculée fournit un simplificateur normal pour la relation d'équivalence modulo $\mathfrak{p}$ (elle permet de décider de l'appartenance à $\mathfrak{p}$ ). Elle permet aussi de calculer des développements de Taylor de solutions de $\Sigma$. L'algorithme est implanté dans un paquetage MAPLE**
\end{abstract}

\begin{abstract}
This paper deals with systems of polynomial differential equations, ordinary or with partial derivatives. The embedding theory is the differential algebra of Ritt and Kolchin. We describe an algorithm, named Rosenfeld-Gröbner, which computes a representation for the radical $\mathfrak{p}$ of the differential ideal generated by any such system $\Sigma$. The computed representation constitutes a normal simplifier for the equivalence relation modulo $\mathfrak{p}$ (it permits to test membership in $\mathfrak{p}$ ). It permits also to compute Taylor expansions of solutions of $\Sigma$. The algorithm is implemented within a package ${ }^{* * *}$ in MAPLE.
\end{abstract}

\footnotetext{
* A part of this work (in particular the MAPLE diffalg package) was realized while the first author was a postdoctoral fellow at the Symbolic Computation Group of the University of Waterloo, N2L 3G6 Ontario, Canada.

** Ce paquetage (diffalg) est disponible. dans la bibliothèque standard de Maple depuis Maple VR5.

*** The package (diffalg) is available in Maple standard library since Maple VR5.

F. Boulier, M. Petitot: Université Lille I, LIFL, 59655 Villeneuve d'Ascq, France, email \{Francois.Boulier,Michel.Petitot\}@lifl.fr

D. Lazard: Université Paris VI, LIP6, 75252 Paris, France, email Daniel.Lazard@lip6.fr

F. Ollivier: École polytechnique, LIX, 91128 Palaiseau CEDEX, France, email Francois.Ollivier@lix.polytechnique.fr
} 


\section{Introduction}

The following system $\Sigma$ (which has no physical significance) is a system of three polynomial differential equations with partial derivatives.

$$
\Sigma\left\{\begin{array}{l}
\left(\frac{\partial}{\partial x} u(x, y)\right)^{2}-4 u(x, y)=0 \\
\left(\frac{\partial^{2}}{\partial x \partial y} u(x, y)\right)\left(\frac{\partial}{\partial y} v(x, y)\right)-u(x, y)+1=0 \\
\frac{\partial^{2}}{\partial x^{2}} v(x, y)-\frac{\partial}{\partial x} u(x, y)=0
\end{array}\right.
$$

In the following, we denote (for short) derivations using indices. The system $\Sigma$ becomes

$$
\Sigma\left\{\begin{array}{l}
u_{x}^{2}-4 u=0, \\
u_{x y} v_{y}-u+1=0, \\
v_{x x}-u_{x}=0
\end{array}\right.
$$

The Rosenfeld-Gröbner algorithm that we present in this paper computes a representation of the radical $\mathfrak{p}$ of the differential ideal ${ }^{1}$ generated by $\Sigma$. This representation tells us in particular that the solutions of $\Sigma$ (which turn out to be polynomials) depend on three arbitrary constants and permits us to compute Taylor expansions of these solutions. If we expand them in the neighborhood of the origin then the arbitrary constants are $u(0,0), v(0,0)$ and $v_{x}(0,0)$. For $u(0,0)=5, v(0,0)=421$ and $v_{x}(0,0)=\pi$ our algorithm gives us (computations are detailed in section 8)

$$
\begin{aligned}
u(x, y)= & 5+x \sqrt{10} \sqrt{2}+y \sqrt{10}+x^{2}+x y \sqrt{2}+\frac{1}{2} y^{2} \\
v(x, y)= & 421+x \pi+2 y \sqrt{2}+\frac{1}{2} x^{2} \sqrt{10} \sqrt{2}+x y \sqrt{10}+ \\
& \frac{1}{4} y^{2} \sqrt{10} \sqrt{2}+\frac{1}{3} x^{3}+\frac{1}{2} x^{2} y \sqrt{2}+\frac{1}{2} x y^{2}+\frac{1}{12} y^{3} \sqrt{2} .
\end{aligned}
$$

The applied mathematical theory is called differential algebra. It was initiated mostly by French and American researchers at the early twentieth century (Riquier [1910], Janet [1920] and [1929] and Ritt [1932]) and really developed by the American teams of Ritt [1950] and Kolchin [1973]. Differential algebra aims at studying differential equations from a purely algebraic point of view. It is much closer to ordinary commutative algebra

\footnotetext{
${ }^{1}$ We make precise in further sections some of the notations and the terminology used in this introduction
} 
than to analysis. The Rosenfeld-Gröbner algorithm represents the radical of the differential ideal generated by any finite system $\Sigma$ of polynomial differential equations as a finite intersection of differential ideals $\mathfrak{r}$ 's (that we call regular).

$$
\mathfrak{p}=\sqrt{[\Sigma]}=\mathfrak{r}_{1} \cap \cdots \cap \mathfrak{r}_{n} .
$$

Each regular differential ideal $\mathfrak{r}_{i}$ is presented by a set of differential polynomial equations $C_{i}$ which satisfies:

1. $C_{i}$ is a canonical representative of $\mathfrak{r}_{i}$,

2. $C_{i}$ reduces to zero a differential polynomial $p$ if and only if $p \in \mathfrak{r}_{i}$.

Therefore, the set of the C's constitutes a normal simplifier for the equivalence relation modulo $\mathfrak{p}$ (i.e. an algorithm which decides membership in $\mathfrak{p}$ ). The simplifier is not canonical for the representation may contain redundant components: every differential prime ideal which is minimal over $\mathfrak{p}$ is minimal over at least one of the regular differential ideals produced but the converse is not true. Assume the solutions of $\mathfrak{p}$ depend on finitely many arbitrary constants. The algorithm separates the solutions which do not depend on the same number of arbitrary constants. In our introductory example, only one regular differential ideal was produced. This proves that all the solutions of $\mathfrak{p}$ depend on three arbitrary constants. An implementation of this algorithm was realized for the MAPLE computer algebra software. It is embedded in a package named diffalg.

\section{Used theorems}

The Rosenfeld-Gröbner algorithm relies mainly on three theorems:

1. a theorem of zeros (Hilbert's Nullstellensatz), which states that a polynomial $p$ belongs to the radical of an ideal presented by a basis $\Sigma$ if and only if every solution of $\Sigma$ is a solution of $p$; we apply this theorem in the algebraic and in the differential case,

2. a lemma of Rosenfeld, which gives a sufficient condition so that a system of polynomial differential equations admits a solution if and only if this same system, considered as a purely algebraic system admits a solution,

3. a lemma of Lazard, which establishes that each regular ideal $\mathfrak{r}$ is radical and that all its prime components have a same parametric set (this property is stronger than "defining an unmixed algebraic variety"). 
It utilizes only the operations and the equality test with zero in the base field of the equations: we refer to Ritt's reduction algorithms, computations of Gröbner bases and splittings similar to those in the elimination methods of Seidenberg [1956]. In particular, it does not need any factorization.

\section{New results}

The Rosenfeld-Gröbner algorithm was first described by Boulier [1994] and improved by Boulier et al. [1995]. This paper contains new results. We give in section 2 a proof of Lazard's Lemma which is more precise than the one we gave in [Boulier et al., 1995, Lemma 2, page 161]. We give an original presentation of the fundamental Rosenfeld's Lemma. We present it as a property of some class of systems of polynomial differential equations and inequations instead of a property of some class of sets of differential polynomials. We give a version of Rosenfeld's Lemma more general than the one of Rosenfeld (this was already proved by Boulier [1997]) and not contained in Kolchin's version. Briefly, our version only imposes to the ideals to be saturated by the separants of the differential polynomials (and no more by the initials). It also only imposes to the set of equations to be triangular instead of autoreduced (but this is anecdotic). Since Lazard's Lemma also holds in such a situation, we formulate our theorems without considering the initials of differential polynomials (though we do it in our implementation for efficiency reasons). This is an improvement w.r.t. Kolchin's theory. We prove new results for regular ideals: Theorems 25 and 54 . The former permits to compute the minimal differential prime components of regular ideals and provides also informations about these prime ideals without having to compute them ; the latter gives us an original presentation of a well known proof about formal power series. The algorithm presented is much more efficient than the one of 1995. It applies for polynomial differential equations an analogue of the second criterion proved by Buchberger [1979] for Gröbner bases. Our implementation of this criterion was designed after the method of Gebauer and Möller [1988].

\section{Comparison with other methods}

There is a strong relationship between our algorithm and Seidenberg's work. Seidenberg [1956] designed elimination algorithms for systems of ODE and PDE in characteristic zero and non zero. His PDE elimination algorithm in characteristic zero actually solves the same problem we are solving: deciding membership in the radical of a finitely generated differential ideal. He proved (Theorem 6, page 51) an analogue of Rosenfeld's Lemma which is a 
bit weaker (restriction to orderly rankings on the derivatives of a single differential indeterminate) and more technical (note Rosenfeld [1959] presents his Lemma as a new version of Seidenberg's Theorem). In his Theorem 11, page 59 he shows that, if $\Sigma$ is a system which satisfies the hypotheses of his Theorem 6 then every algebraic solution of $\Sigma$ furnishes a unique differential solution. He showed later [Seidenberg, 1969] how differential solutions can be converted as formal power series. There are differences between Seidenberg's algorithm and ours. The most important is the following: the Rosenfeld-Gröbner algorithm computes a representation of radical differential ideals which can be used afterwards for testing membership in the ideal many times afterwards while Seidenberg's decides if a differential polynomial $p$ belongs to the radical of the differential ideal generated by a finite family $\Sigma$ by eliminating successively all the differential indeterminates which occur in the system $\Sigma=0, p \neq 0$ in order to test if this system admits solutions (Hilbert's theorem of zeros). The answer of his algorithm is a boolean. Another important difference: Seidenberg's elimination algorithms are restricted to elimination rankings between differential indeterminates which induce very explosive computations, while orderly rankings are handled by the Rosenfeld-Gröbner algorithm (this is the case for instance in our introductory example). Ritt [1950] gave a method to decompose the radical of an ordinary differential ideal as an intersection of prime differential ideals, providing a characteristic set for each of these ideals. This decomposition is not the minimal one because of the redundancy problem (still open). That algorithm is inconvenient because it is only partially effective: it proceeds by factorization over a tower of algebraic field extensions of the field of coefficients. To our knowledge, it has not been implemented. It only applies for ODE. Wu [1989] designed a variant of Ritt's algorithm for ordinary differential equations, with a notion of characteristic set weaker than Ritt's (e.g. a characteristic set in the sense of Wu may have no solution). Other authors (e.g. Wang [1994]) developed later Wu's and Seidenberg's ideas. These algorithms only apply for ODE. Ollivier [1990] and Carra-Ferro [1987] have independently tried to generalize Gröbner bases to systems of ordinary polynomial differential equations. These differential Gröbner bases are in general infinite, even for ODE systems. Another definition of differential Gröbner bases was attempted by Mansfield [1991]. The algorithm DIFFGBASIS, implemented in MAPLE, utilizes Ritt's algorithm of reduction and then always terminates. It handles PDE systems. In general however, it cannot guarantee its output to be a differential Gröbner basis. Note that the membership problem in an arbitrary differential ideal is undecidable [Gallo et al., 1991], and the membership problem of a finitely generated differential ideal is still open. Bouziane et al. [2001] and Maârouf [1996] designed recently a variant 
of the Rosenfeld-Gröbner algorithm. They started from the algorithm of Kalkbrener [1993] which compute decompositions of radicals of ideals in non differential polynomial algebras. They describe a method for computing characteristic sets of prime differential ideals different from our methods given in [Ollivier, 1990], [Boulier, 1994] and [Boulier et al., 1995, section 5, page 164]. Reid et al. [1996] and Reid et al. [2001] developed algorithms for studying systems of PDE and computing Taylor expansions of their solutions. These methods are based more on differential geometry than on algebra. They do not claim to be as general as the Rosenfeld-Gröbner algorithm.

\section{Organization of the paper}

Sections 1 and 2 deal with commutative algebra. The former contains preliminaries; in the latter, we prove Lazard's Lemma and show how some computations can be performed in dimension zero. Section 3 contains differential algebra preliminaries. In section 4 we prove our version of Rosenfeld's Lemma and some technical results which will be used for efficiently testing the coherence hypothesis of this lemma (in particular, we show there our analogue of Buchberger's second criterion). Section 5 shows how to represents radical differential ideals as intersections of regular differential ideals. This is the core of the Rosenfeld-Gröbner algorithm. In the next section, we show how to compute canonical representatives for regular differential ideals and we state the Rosenfeld-Gröbner algorithm as a theorem (Theorem 60) with an effective proof. The algorithm is obtained by translating the proof in any programming language. In section 7 we explain how algebraic solutions of regular differential ideals can be expanded as formal power series. A few examples are developed in the last section.

\section{Commutative algebra preliminaries}

Let $R=K[X]$ be a polynomial ring where $K$ is a field and $X$ is an alphabet (possibly infinite) endowed with an ordering $\mathscr{R}$. Let $p \in R \backslash K$ be a polynomial. The leader of $p$ is the greatest indeterminate $x \in X$ w.r.t. $\mathscr{R}$ which appears in $p$. It is denoted by $\operatorname{ld} p$. Let $d=\operatorname{deg}(p, x)$ be the degree of $p$ in $x$. The initial $i_{p}$ of $p$ is the coefficient of $x^{d}$ in $p$. The separant $s_{p}$ of $p$ is the polynomial $\partial p / \partial x$. The rank of $p$ is the monomial $x^{d}$. It is denoted by rank $p$. The rank of a set of polynomials is the set of ranks of the elements of the set. If $A \subset R \backslash K$ is a set of polynomials then $I_{A}$ (respectively $S_{A}$ ) denotes the set of the initials (respectively separants) of the elements of $A$ and $H_{A}=I_{A} \cup S_{A}$. If $p$ and $q$ are two polynomials with ranks $x^{d}$ and $y^{e}$ then $q<p$ if $y<x$ 
or $y=x$ and $e<d$. Let $A=\left\{p_{1}, \ldots, p_{n}\right\}$ and $A^{\prime}=\left\{p_{1}^{\prime}, \ldots, p_{n^{\prime}}^{\prime}\right\}$ be two nonempty subsets of $R \backslash K$. Renaming the polynomials if needed, assume $\operatorname{rank} p_{i} \leq \operatorname{rank} p_{i+1}$ and $\operatorname{rank} p_{j}^{\prime} \leq \operatorname{rank} p_{j+1}^{\prime}$ for all $i<n, j<n^{\prime}$. The set $A$ is said to be of lower rank than $A^{\prime}$ if there exists some $i \leq \min \left(n, n^{\prime}\right)$ such that $p_{i}<p_{i}^{\prime}$ and $\operatorname{rank} p_{j}=\operatorname{rank} p_{j}^{\prime}$ for $1 \leq j<i$ else if $n>n^{\prime}$ and $\operatorname{rank} p_{j}=\operatorname{rank} p_{j}^{\prime}$ for $1 \leq j \leq n^{\prime}$. Two sets of polynomials such that none of them is of lower rank than the other one are said to have the same rank. A subset $A$ of $R \backslash K$ is said to be triangular if the leaders of its elements are pairwise different.

If $A \subset R$ then $(A)$ denotes the smallest ideal of $R$ containing $A$. If $\mathfrak{a}$ is an ideal of $R$ then the radical $\sqrt{\mathfrak{a}}$ of $\mathfrak{a}$ is the ideal of all the elements of $R$, a power of which lies in $\mathfrak{a}$. An ideal equal to its radical is said to be radical. Any radical ideal $\mathfrak{r}$ of a polynomial ring $R=K[X]$ ( $X$ finite) is a finite intersection of prime ideals which is unique when minimal. A component (say $\mathfrak{p}_{1}$ ) of an intersection $\mathfrak{r}=\mathfrak{p}_{1} \cap \cdots \cap \mathfrak{p}_{n}$ is said to be redundant w.r.t. $\mathfrak{r}$ if $\mathfrak{r}=\mathfrak{p}_{2} \cap \cdots \cap \mathfrak{p}_{n}$. An element $p$ of a ring $R$ is said to be a divisor of zero if $p \neq 0$ and there exists in $R$ an element $q \neq 0$ such that the product $p q=0$. If $\mathfrak{r}$ is an ideal and $S$ is a finite subset of a ring $R$ then the saturation $\mathfrak{r}: S^{\infty}$ of $\mathfrak{r}$ by $S$ is the ideal of all the polynomials $p \in R$ such that there exists a power product $h$ of elements of $S$ such that $h p \in \mathfrak{r}$.

\subsection{Gröbner bases}

In this section $R=K[X]$ denotes a polynomial ring over a field. We only recall some properties of Gröbner bases. Reference books are those of Cox et al. [1992] Becker and Weispfenning [1991]. If $B$ is a Gröbner basis of an ideal $\mathfrak{r}$ of a polynomial ring $R=K[X]$ for an ordering $\mathscr{R}$. The reduction by $B$, denoted by $\underset{B}{\stackrel{*}{\longrightarrow}}$ preserves the equivalence relation $\bmod \mathfrak{r}$ and we have

1. $\mathfrak{r}=(B)$,

2. when it is reduced, a Gröbner basis is a canonical representative of $\mathfrak{r}$ in the sense that it only depends on the ideal and on the ordering [Cox et al., 1992, chapter 2, §7, Proposition 6],

3. the ideal $\mathfrak{r}$ is equal to $R$ if and only if $1 \in B$ [Becker and Weispfenning, 1991, Corollary 6.16],

4. given any $p \in R$, there exists a unique polynomial $\bar{p}$ irreducible by $B$ such that $p \underset{B}{\stackrel{*}{\longrightarrow}} \bar{p}$. This polynomial is a canonical representative of the residue class of $p$ modulo $\mathfrak{r}$ (it only depends on the ideal and the 
ordering). In particular, if $p \in \mathfrak{r}$ then $\bar{p}=0$ [Cox et al., 1992, chapter 2, $\S 6$, Proposition 1 and Corollary 1],

Even if $X$ is infinite, one can compute Gröbner bases of finitely generated ideals of $K[X]$. This remark is important since we are going to compute Gröbner bases of (non differential) ideals in differential polynomial rings. The theoretical justification is given by the following lemma.

Lemma 1. - Let $\mathfrak{r}$ be an ideal of a ring $R$ and $x$ be transcendental over $R$. If $\phi$ denotes the canonical ring homomorphism $\phi: R \rightarrow R[x]$ then $\phi^{-1}(\phi \mathfrak{r})=\mathfrak{r}$.

Let $A=\left\{p_{1}, \ldots, p_{n}\right\}$ and $S=\left\{s_{1}, \ldots, s_{m}\right\}$ be finite sets of polynomials of $R$. Let $\left\{z_{1}, \ldots, z_{m}\right\}$ be a finite set of indeterminates over $R$. One gets a Gröbner basis $B_{0}$ of the ideal $S^{-1}(A)$ of $S^{-1} R$ by computing a Gröbner basis of the set

$$
\left\{p_{1}, \ldots, p_{n}, s_{1} z_{1}-1, \ldots, s_{m} z_{m}-1\right\}
$$

for any ordering [Eisenbud, 1995, exercise 2.2, page 79]. Each $z_{i}$ stands for $1 / s_{i}$. To get a Gröbner basis $B_{1}$ of $(A): S^{\infty}$, compute first $B_{0}$ for any ordering which eliminates the $z$ 's. Then $B_{1}=B_{0} \cap R$ [Becker and Weispfenning, 1991, Proposition 6.15].

\section{Lazard's Lemma}

Lazard's Lemma (Theorem 3) is a result of commutative algebra, interesting in itself. It was first published in [Boulier et al., 1995, Lemma 2, page 161] with a proof relying on basic arguments. During the Special Year in Differential Algebra and Algebraic Geometry organized in 1995 at the City College of New York by Prof. Hoobler and Sit, a weakness in the proof was pointed out ${ }^{2}$ : there was a claim which was true but not proved. Morrison [1995] proved then a generalized version of the lemma which is presented in [Morrison, 1999]. Another proof was written later by Schicho and Li [1995]. The one we give here only relies on elementary commutative algebra [Zariski and Samuel, 1958, chapter IV]. In this sense, it is simpler than the other ones. The knowledge of Morrison's proof helped us to fix ours. Section 2.2 contains the argument [Ollivier, 1998] missing in [Boulier et al., 1995].

DEFINITION 2. - (regular algebraic systems)

A system $A=0, S \neq 0$ of a polynomial ring $R$ is said to be a regular algebraic system (for an ordering $\mathscr{R}$ ) if

1. A is triangular,

\footnotetext{
${ }^{2}$ The first author would like to thank Prof. Hoobler, Sit and in particular Prof. Sally Morrison for many fruitful comments and email communications.
} 


\section{2. $S$ contains the separants of the elements of $A$.}

The ideal $(A): S^{\infty}$ is called the regular algebraic ideal defined by the system. The system is said to be inconsistent if $(A): S^{\infty}=R$. It is said to be consistent otherwise.

THEOREM 3. - (Lazard's Lemma)

Let $A=0, S \neq 0$ be a consistent regular algebraic system of a polynomial ring $R=K[X]$. Denote $L$ the set of the leaders of the elements of $A$ and $N=X \backslash L$. Then

1. the regular algebraic ideal $(A): S^{\infty}$ is radical,

2. if $\mathfrak{p}$ is a prime ideal minimal over $(A): S^{\infty}$ then $\operatorname{dim} \mathfrak{p}=|N|$ and $\mathfrak{p} \cap K[N]=(0)$.

Proof. - Notice it is enough to prove the theorem in the case $S=S_{A}$ for, if $(A): S_{A}^{\infty}$ is radical, the ideal $(A): S^{\infty}$ is the intersection of the prime ideals which are minimal over $(A): S_{A}^{\infty}$ and which do not meet $S$ [Zariski and Samuel, 1958, chapter IV, §10, Theorem 17]. Propositions 9 and 10 imply that if $\mathfrak{p}$ is an associated prime of $(A): S_{A}^{\infty}$ then $\operatorname{dim} \mathfrak{p}=|N|$ and $\mathfrak{p} \cap K[N]=(0)$. This proves the point 2 .

The nonzero elements of $K[N]$ are thus different from zero and do not divide zero in $R /(A): S_{A}^{\infty}$. The elements of $S_{A}$ are not zero and do not divide zero either in $R /(A): S_{A}^{\infty}$. Thus the total rings of fractions [Zariski and Samuel, 1958, chapter I, §19] of $R /(A): S_{A}^{\infty}$ and $S_{\bar{A}}^{-1} \bar{R} / S_{\bar{A}}^{-1}(\bar{A})$ are isomorphic, where $\bar{R}=K(N)[L]$ and $\bar{A}$ denotes the image of $A$ by the canonical ring homorphism $R \rightarrow \bar{R}$. A product of fields is isomorphic to its own total ring of fractions [Zariski and Samuel, 1958, chapter I, $\$ 19$, Corollary 1] thus, by Proposition 6 , the total ring of fractions of $R /(A): S_{A}^{\infty}$ is isomorphic to a product of fields. A product of fields does not involve any nilpotent [Zariski and Samuel, 1958, chapter III, §7, page 148] element thus $R /(A): S_{A}^{\infty}$ does not either whence $(A): S_{A}^{\infty}$ is radical.

In the sequel, we consider the ideal $(A): S_{A}^{\infty}$. We denote by $L$ the set of leaders of the triangular set $A$ and $N$ the remaining indeterminates. Thus $(A): S_{A}^{\infty} \subset R=K[N, L]$. We assume $(A): S_{A}^{\infty} \neq R$.

\subsection{Lazard's Lemma in dimension zero}

In this section we consider the case $|N|=0$.

We denote by $S_{A}^{-1} R$ the ring localized at $S_{A}$ and $S_{A}^{-1}(A)$ the ideal generated by the image of $(A)$ in $S_{A}^{-1} R$ [Eisenbud, 1995, section 2]. 
Lemma 4. - Let $K[x]$ be a polynomial ring in one indeterminate over a field. Let $p \in K[x]$ be a polynomial and $s$ be its separant. The ideal $s^{-1} K[x] / s^{-1}(p)$ is isomorphic to a product of algebraic field extensions of $K$.

Proof. - The ideal $s^{-1}(p)$ is generated by the product of the irreducible simple factors of $p$. These factors generate comaximal ideals in $K[x]$. The lemma comes from the Chinese remainders Theorem [Eisenbud, 1995, section 2, exercise page 79].

Lemma 5. - If $R_{0}$ is a ring isomorphic to a product of algebraic field extensions of $K$ and $x$ is a new inderminate, $p \in R_{0}[x]$ is a polynomial and $s=\partial p / \partial x$ is its separant then $s^{-1} R_{0}[x] / s^{-1}(p)$ is isomorphic to a product of algebraic field extensions of $K$.

Proof. - Let $R_{0} \simeq K_{1} \times \cdots \times K_{h}$. We have $R_{0}[x] \simeq K_{1}[x] \times \cdots \times K_{h}[x]$. Denote $\pi_{i}$ the canonical ring homomorphism $R_{0}[x] \rightarrow K_{i}[x](1 \leq i \leq h)$. We have

$$
s^{-1} R_{0}[x] / s^{-1}(p) \simeq \prod_{i=1}^{h}\left(\pi_{i} s\right)^{-1} K_{i}[x] /\left(\pi_{i} s\right)^{-1}\left(\pi_{i} p\right) .
$$

Since $\pi_{i} s=\partial \pi_{i} p / \partial x(1 \leq i \leq h)$, Lemma 4 applies therefore each term $\left(\pi_{i} s\right)^{-1} K_{i}[x] /\left(\pi_{i} s\right)^{-1}\left(\pi_{i} p\right)$ is isomorphic to a product of algebraic field extensions of $K$ thus so is $s^{-1} R_{0}[x] / s^{-1}(p)$.

Proposition 6. - The ring $S_{A}^{-1} R / S_{A}^{-1}(A)$ is isomorphic to a product of algebraic field extensions of $K$.

Proof. - Apply Lemma 5 inductively on $|A|$.

\subsection{Non leaders form a parametric set}

If $\mathfrak{i}$ and $\mathfrak{j}$ are two ideals of $R$ then the quotient $\mathfrak{i}: \mathfrak{j}$ of $\mathfrak{i}$ by $\mathfrak{j}$ [Zariski and Samuel, 1958, chapter III, §7] is defined by $\mathfrak{i}: \mathfrak{j}=\{p \in R \mid \forall q \in \mathfrak{j}, p q \in \mathfrak{i}\}$

Lemma 7. - Denote $\mathfrak{i}=(A): S_{A}^{\infty}$. If $h \in R$ then for every $q \in \mathfrak{i}:(h)$ we have

$$
\forall x \in L, \frac{\partial q}{\partial x}=0 \quad \Rightarrow \quad \forall x \in N, \frac{\partial q}{\partial x} \in \mathfrak{i}:(h) .
$$

Proof. - Denote $D$ the determinant of the jacobian matrix $J=$ $(\partial p / \partial x)_{p \in A, x \in L}$ of $A$, which is the product of the elements of $S_{A}$ since $A$ is triangular. Assume $q \in \mathfrak{i}:(h)$. Then there exists some $\alpha \geq 0$ and some $m_{p} \in R$ $(p \in A)$ such that

$$
D^{\alpha} h q=\sum_{p \in A} m_{p} p
$$


Assume $x \in L$. Differentiate relation (1) w.r.t. $x$, multiply it by $D$ and $h$ and apply the fact that $\partial q / \partial x=0$. We conclude that

$$
D h \sum_{p \in A} m_{p} \frac{\partial p}{\partial x} \in(A) .
$$

Denote $\tilde{J}$ the cofactors matrix of $J$ and $I$ the identity matrix. Using the fact that $J \tilde{J}=D I$ we find that $D^{2} h m_{p} \in(A)$ for each $p \in A$ which implies $m_{p} \in \mathfrak{i}:(h)$ for each $p \in A$. Differentiate relation (1) w.r.t. some $x \in N$ now, multiply by $D$ and $h$ and simplify. We conclude that $\partial q / \partial x \in \mathfrak{i}:(h)$.

Corollary 8. - Denote $\mathfrak{i}=(A): S_{A}^{\infty}$. If $h \in R$ is a polynomial such that $\mathfrak{i}:(h) \neq R$ then $\mathfrak{i}:(h) \cap K[N]=(0)$.

Proof. - If $q \in \mathfrak{i}:(h) \cap K[N]$ then for any $x \in L$ we have $\partial q / \partial x=0$. Using Lemma 7 we see $\mathfrak{i}:(h) \cap K[N]$ is stable under the action of the partial derivations w.r.t. all the indeterminates. This ideal is therefore either equal to $K[N]$ (in which case $\mathfrak{i}:(h)=R$ ) or to $(0)$.

Proposition 9. - If $\mathfrak{q}$ is an isolated primary component of $(A): S_{A}^{\infty}$ then $\operatorname{dim} \mathfrak{q}=|N|$ and $\mathfrak{q} \cap K[N]=(0)$.

Proof. - Let $h \in R$ be a polynomial belonging to all the associated primes of $\mathfrak{i}=(A): S_{A}^{\infty}$ but not to the associated prime of $\mathfrak{q}$. This polynomial exists for $\mathfrak{q}$ is isolated. For $\beta \geq 0$ great enough, $h^{\beta}$ belongs to all the primary components of $\mathfrak{i}$ but not to $\mathfrak{q}$ and we have $\mathfrak{i}:\left(h^{\beta}\right)=\mathfrak{q}$. Corollary 8 implies $\mathfrak{q} \cap K[N]=(0)$ whence $\operatorname{dim} \mathfrak{q} \geq|N|$.

Now, let $\bar{R}=K(N)[L]$ and $\bar{A}$ be the image of $A$ by the canonical ring homorphism $\phi: R \rightarrow \bar{R}$. Let $\mathfrak{p}$ be a prime ideal minimal over $\mathfrak{i}$. This ideal does not meet the multiplicative family of the nonzero elements of $K[N]$. By [Zariski and Samuel, 1958, chapter IV, $\S 10$, Theorem 17], the prime ideal $(\phi \mathfrak{p})$ is minimal over the ideal $\mathfrak{j}=(\bar{A}): S_{\bar{A}}^{\infty}$. Proposition 6 implies that $\operatorname{dim} \overline{\mathfrak{p}}=0$ whence $\operatorname{dim} \mathfrak{p}=|N|$.

Proposition 10. - If $\mathfrak{q}$ is a primary component of $(A): S_{A}^{\infty}$ then $\mathfrak{q}$ is isolated.

Proof. - Assume $\mathfrak{i}=(A): S_{A}^{\infty}$ admits an imbedded primary component. Proposition 9 implies that $\operatorname{dim} \mathfrak{q}<|N|$ whence $\mathfrak{q} \cap K[N] \neq(0)$ for each imbedded primary component $\mathfrak{q}$ of $\mathfrak{i}$. Let $h$ be any polynomial, taken in all the isolated components of $\mathfrak{i}$, such that $h \notin \mathfrak{i}$. The ideal $\mathfrak{i}:(h)$ is different from $R$. It is equal to the intersection of the ideals $\mathfrak{q}_{i}:(h)$ for all the imbedded primary components $\mathfrak{q}_{i}$ of $\mathfrak{i}$. Thus it contains the intersection of the imbedded primary components of $\mathfrak{i}$. Thus $\mathfrak{i}:(h) \cap K[N] \neq(0)$. This contradiction to Corollary 8 proves the proposition. 
DeFINITION 11. - (associated Gröbner basis)

Let $A=0, S \neq 0$ be a consistent regular algebraic system of a ring $R=K[X]$. Denote $L$ the set of the leaders of the elements of $A$ and $N=$ $X \backslash L$. The reduced Gröbner basis of the ideal $(A): S^{\infty}$, computed in the ring $K(N)[L]$ for the elimination ordering given by the ordering over $X$ is called the Gröbner basis associated to $A=0, S \neq 0$.

Corollary 12. - If $A=0, S \neq 0$ is a consistent regular algebraic system of a polynomial ring $R=K[X]$ and $x_{i} \in X$ is an indeterminate then the following conditions are equivalent

1. $x_{i}$ is the leader of some element of $A$,

2. $x_{i}$ is the leader of some element of the Gröbner basis associated to the system $A=0, S \neq 0$,

3. $x_{i}$ is the leader of any characteristic set (for the ordering defined over $X$ ) of any prime ideal minimal over $(A): S^{\infty}$.

\subsection{Computing in dimension zero}

Let $A=0, S \neq 0$ be a regular algebraic system of a polynomial ring $R_{0}=$ $K[X]$ for an ordering $\mathcal{R}$. Let $L \subset X$ be the set of the leaders of the elements of $A$ and $N=X \backslash L$. Denote $\mathfrak{r}_{0}=(A): S^{\infty}$ and $B_{0}$ the reduced Gröbner basis of $\mathfrak{r}_{0}$ w.r.t. the elimination ordering given by $\mathcal{R}$. Let $R_{1}=K(N)[L]$ be the polynomial ring obtained by extending the ground field $K$ with $N$ and $\varphi$ the canonical ring homomorphism $R_{0} \longrightarrow R_{1}$. Denote $\mathfrak{r}_{1}=(\varphi A)$ : $(\varphi S)^{\infty}$ and $B_{1}$ the Gröbner basis associated to $A=0, S \neq 0$. The basis $B_{1}$ is a Gröbner basis of $\mathfrak{r}_{1}$. Because of Theorem 3 the ring homomorphism $R_{0} / \mathfrak{r}_{0} \longrightarrow R_{1} / \mathfrak{r}_{1}$ is injective (by [Zariski and Samuel, 1958, chapter I, $\S 19$ ] and the fact that the nonzero elements of $K[N]$ are regular modulo $\mathfrak{r}_{0}$ [Zariski and Samuel, 1958, chapter IV, §6, Corollary 3]) and there is a one-to-one correspondence between the prime ideals $\mathfrak{p}_{1}, \ldots, \mathfrak{p}_{m}$ which are minimal over $\mathfrak{r}_{0}$ and the prime ideals $\mathfrak{q}_{1}, \ldots, \mathfrak{q}_{m}$ which are minimal over $\mathfrak{r}_{1}$ [Zariski and Samuel, 1958, chapter IV, $\S 10$, Theorem 17]. The q's are dimension zero ideals thus so is $\mathfrak{r}_{1}$. Therefore, though $B_{1}$ is not a Gröbner basis of $\mathfrak{r}_{0}$, many computations can be performed using the latter since

1. for any $p \in R_{0}$ we have $p \in \mathfrak{r}_{0}$ if and only if $\varphi p \underset{B_{1}}{\stackrel{*}{\longrightarrow}} 0$,

2. a polynomial $p \in R_{0}$ is a divisor of zero modulo $\mathfrak{r}_{0}$ if and only if $\varphi p$ is a divisor of zero modulo $\mathfrak{r}_{1}$, 
3. minimal triangular subsets of $B_{0}$ have the same rank as minimal triangular subsets of $B_{1}$.

The basis $B_{1}$ turns out to be much smaller and faster to compute than $B_{0}$. It is sometimes faster to compute Gröbner bases of regular algebraic ideals in dimension zero in MAPLE than to compute the Gröbner bases in positive dimension using the GB software of Faugère (which runs usually one thousand times faster than the MAPLE's implementation of the Buchberger's algorithm).

\section{Differential algebra preliminaries}

The reference book is the one of [Kolchin, 1973, chapters I-IV]. Readers who discover the theory had probably better however to start with the book of Ritt [1950]. A differential ring is a ring endowed with $m$ derivations $\delta_{1}, \ldots, \delta_{m}$ which commute pairwise. Derivation operators are denoted multiplicatively $\theta=\delta_{1}^{a_{1}} \cdots \delta_{m}^{a_{m}}$ where the $a$ 's are nonnegative integers. The sum of the exponents $a$ 's is the order of $\theta$, denoted by ord $\theta$. The identity operator has order 0 . All other operators are said to be proper. If $\theta=\delta_{1}^{a_{1}} \cdots \delta_{m}^{a_{m}}$ and $\phi=\delta_{1}^{b_{1}} \cdots \delta_{m}^{b_{m}}$ then $\theta \phi=\delta_{1}^{a_{1}+b_{1}} \cdots \delta_{m}^{a_{m}+b_{m}}$. If $a_{i} \geq b_{i}$ for $i=1, \ldots, m$ then $(\theta / \phi)=\delta_{1}^{a_{1}-b_{1}} \cdots \delta_{m}^{a_{m}-b_{m}}$. The monoid of derivation operators is denoted by $\Theta$. If $R$ is a differential ring and $S \subset R$ then $\Theta S$ denotes the smallest subset of $R$ containing $S$ and stable under derivation. If $R_{0} \subset R_{1}$ are two differential rings and $S \subset R_{1}$ then $R_{0}\{S\}$ denotes the smallest differential ring containing $R_{0}$ and $S$ i.e. $R_{0}[\Theta S]$. We deal with a differential polynomial $\operatorname{ring} R=$ $K\left\{u_{1}, \ldots, u_{n}\right\}$ where $K$ is a differential field of characteristic zero. The $u$ 's are called differential indeterminates and the $\theta u$ 's are called derivatives. The set of the derivatives is denoted by $\Theta U$. The differential ring $R$ can be viewed as a non differential polynomial ring $K[\Theta U]$ whose indeterminates are the derivatives of $R$. The definitions given for non differential polynomial rings hold therefore for differential ones. If $\theta u$ and $\phi u$ are derivatives of some same differential indeterminate $u$, we denote by $\operatorname{lcd}(\theta u, \phi u)$ the least common derivative between $\theta u$ and $\phi u$. It is equal to $\operatorname{lcm}(\theta, \phi) u$. A ranking $\mathcal{R}$ is an ordering over $\Theta U$ compatible with the action of the derivations over $\Theta U$ [Kolchin, 1973, chapter I, §8]:

1. $\delta v>v$ (for all derivation $\delta$ and $v \in \Theta U$ ),

2. $v>w \Rightarrow \delta v>\delta w$ (for all derivation $\delta$ and $v, w \in \Theta U$ ).

Rankings such that $\operatorname{ord} \theta>\operatorname{ord} \phi \Rightarrow \theta v>\phi w$ (for all derivations operators $\theta, \phi$ and all differential indeterminates $v, w)$ are said to be orderly. Rankings 
such that $v>w \Rightarrow \theta v>\phi w$ (for all derivations operators $\theta, \phi$ and all differential indeterminates $v, w$ ) are said to be elimination rankings. Any ranking is a well-ordering [Kolchin, 1973, chapter I, §8]. Properties of rankings imply that the separant of a differential polynomial $p \in R \backslash K$ is also the initial of all the proper derivatives of $p$. If $A \subset R \backslash K$ is a set of differential polynomials and $v$ is any derivative then $A_{v}$ denotes the set of the derivatives of the elements of $A$ whose leaders are less than or equal to $v$ :

$$
A_{v}=\{\theta p \mid p \in A, \theta \in \Theta \text { and } \operatorname{ld} \theta p \leq v\} .
$$

According to this notation, $R_{v}$ denotes the ring of the differential polynomials whose leaders are less than or equal to $v$. Therefore

$$
A \cap R_{v}=\{p \in A \mid \operatorname{ld} p \leq v\} .
$$

Let $p \in R \backslash K$ and $q \in R$ be differential polynomials. Denote rank $p=v^{d}$. The differential polynomial $q$ is said to be partially reduced w.r.t. $p$ if no proper derivative of $v$ appears in $q$; it is said to be reduced w.r.t. $p$ if $q$ is partially reduced w.r.t. $p$ and $\operatorname{deg}(q, v)<d$. A set $A \subset R \backslash K$ is said to be autoreduced if any element of $A$ is reduced w.r.t. any other element of the set.

Definition 13. - $A$ set $A \subset R \backslash K$ is said to be differentially triangular if it is triangular and if its elements are pairwise partially reduced.

Every autoreduced set is finite [Kolchin, 1973, chapter I, §9]. The proof holds also for differentially triangular sets. A characteristic set of a $\operatorname{set}^{3} S \subset$ $R$ is an autoreduced subset of $S$ which has lowest rank among the autoreduced subsets of $S$. It is also a minimal (according to our definition) element in the set of the autoreduced subsets of $S$. If $S \subset R$ admits autoreduced subsets then $S$ admits a characteristic set.

A differential ideal of a differential ring $R$ is an ideal of $R$ stable under differentiation. If $A \subset R$ then $[A]=(\Theta A)$ denotes the smallest differential ideal of $R$ containing $A$. Since $R$ has characteristic zero, the radical of a differential ideal is a differential ideal. Any radical differential ideal $\mathfrak{r}$ of a differential polynomial ring $R$ is a finite intersection of differential prime ideals which is unique when minimal [Kolchin, 1973, chapter III, $\S 4$, Theorem 1] or [Ritt, 1950, chapter I, $\S 16$, Theorem]. The following is a differential analogue of Hilbert's theorem of zeros [Seidenberg, 1952, Nullstellensatz, weak form] or [Kolchin, 1973, chapter IV, §2].

\footnotetext{
${ }^{3}$ This definition corresponds to Ritt's one [Ritt, 1950, I, 5, page 5] and coincides with Kolchin's when $S$ is a differential ideal. Kolchin only defined characteristic sets for ideals [Kolchin, 1973, chapter I, §10, and III, §2].
} 
THEOREM 14. - (theorem of zeros)

Let $R=K\{U\}$ be a differential polynomial ring over a differential field of characteristic zero and $\mathfrak{r}$ be a differential ideal of $R$. A differential polynomial $p$ vanishes on every solution of $\mathfrak{r}$, in any differential field extension of $K$, if and only if $p \in \sqrt{\mathfrak{r}}$.

Proof. - The implication from right to left is immediate. The implication from left to right: if $p \notin \sqrt{\mathfrak{r}}$ then $p$ does not belong to at least one differential prime ideal $\mathfrak{p}$ minimal over $\sqrt{\mathfrak{r}}$. The canonical ring homorphism which maps $R$ to the field of fractions of $R / \mathfrak{p}$ furnishes a solution of $\mathfrak{r}$ which is not a solution of $p$.

COROLlary 15. - A differential polynomial p vanishes on every solution of a system of polynomial differential equations and inequations $A=0, S \neq 0$ iff $p \in \sqrt{[A]: S^{\infty}}$.

PROOF. - Using the definitions of the radical and of the saturation of an ideal, we see that $p \in \sqrt{[A]: S^{\infty}}$ if and only if there exists a product $h$ of elements of $S$ such that $h p \in \sqrt{[A]}$. According to the theorem of zeros, $h p \in \sqrt{[A]}$ if and only if $h p$ vanishes on every solution of the system $A=0$ i.e. if and only if $p$ vanishes on every solution of the system $A=0, S \neq 0$.

The following technical lemma is classical. See [Ritt, 1950, chapter II, $\S 12]$. We are going to use it many times.

Lemma 16. - Let $A$ be a finite subset of some differential polynomial ring $R$. Let $q=s v+r$ be a differential polynomial with leader $v$, such that $\operatorname{deg}(q, v)=$ 1 and $v$ does not appear in $s, r$ nor any element of $A$. For any $p \in R$, if $p \in(A, q)$ and $v$ does not appear in $p$ then $p \in(A): s^{\infty}$.

Proof. - Since $p \in(A, q)$ there exists a formula $(f)$ such that

$$
p=\underbrace{\sum_{p_{i} \in A} B_{i} p_{i}+C q}_{(f)}
$$

where $B_{i}, C \in R$. Apply on the terms of $(f)$ the substitution $v \longrightarrow(q-r) / s$ and multiply by some power of $s$ to clear denominators. Since $v$ does not appear in $p$ and the $p_{i}$ one gets another formula $\left(f^{\prime}\right)$ such that

$$
s^{\alpha} p=\underbrace{\sum_{p_{i} \in A} D_{i} p_{i}+E q}_{\left(f^{\prime}\right)}
$$

where $D_{i}, E \in R$ and $v$ only appears in $q$. Thus $E=0$ and $p \in(A): s^{\infty}$. 


\section{$3.1 \quad$ Ritt's reduction algorithms}

Ritt's reduction algorithms are pseudo-division [Knuth, 1966, vol. 2, page 407] algorithms, extended to differential algebra. Different variants exists [Kolchin, 1973, chapter I, §9] [Ritt, 1950, chapter I, §6]. We fix one of them. Ritt's partial reduction (denoted by partial-rem) is distinguished from Ritt's full reduction (denoted by full-rem).

\section{Specification of the partial reduction algorithm}

If $\bar{q}=q$ partial-rem $A$ denotes the partial remainder of $q$ by $A$ then

1. $\bar{q}$ is partially reduced w.r.t. all the elements of $A$,

2. there exists a power product $h$ of elements of $S_{\bar{A}}$ such that $h q \equiv \bar{q}$ $\left(\bmod \left(\bar{A}_{v}\right)\right)$.

The following instructions provide an algorithm to compute $h$ and $\bar{q}$ from $q$. Build a sequence of pairs $\left(h_{i}, q_{i}\right)$. Initially, set $h_{0}=1$ and $q_{0}=q$ and stop at the first index $n$ such that $q_{n}$ is partially reduced w.r.t. $A$ (then take $h=h_{n}$ and $\left.\bar{q}=q_{n}\right)$. If $i$ is an index such that $q_{i}$ is not partially reduced w.r.t. $A$ then let $w$ be the highest derivative which occurs in $q_{i}$ which is also a proper derivative of the leader of some $p \in A$. If there are many different possibilities for $p$, take any of them. Now, let $\theta$ be the derivation operator such that $\operatorname{ld} \theta p=w$. Take for $q_{i+1}$ the pseudo-remainder of $q_{i}$ by $\theta p$. There exists then some $\alpha \in \mathbb{N}$ such that $s_{p}^{\alpha} q_{i}=q_{i+1} \bmod (\theta p)$. Take $h_{i+1}=s_{p}^{\alpha} h$.

\section{Specification of the full reduction algorithm}

If $\bar{q}=q$ full-rem $A$ denotes the full remainder of $q$ by $A$ then

1. $\bar{q}$ is reduced w.r.t. all the elements of $A$,

2. there exists a power product $h$ of elements of $H_{\bar{A}}$ such that $h q \equiv \bar{q}$ $\left(\bmod \left(\bar{A}_{v}\right)\right)$.

The following instructions provide an algorithm to compute $h$ and $\bar{q}$ from $q$. Build a sequence of pairs $\left(h_{i}, q_{i}\right)$. Initially, set $h_{0}=1$ and $q_{0}=q$ and stop at the first index $n$ such that $q_{n}$ is reduced w.r.t. $A$ (then take $h=h_{n}$ and $\bar{q}=q_{n}$ ). If $i$ is an index such that $q_{i}$ is not reduced w.r.t. $A$ then let $w$ be the highest derivative which occurs in $q_{i}$ s.t. one of the next conditions holds:

1. $w$ is a proper derivative of the leader of some $p \in A$,

2. $w$ is the leader of some $p \in A$ and $\operatorname{deg}\left(q_{i}, w\right) \geq \operatorname{deg}(p, w)$. 
If the first case arises then proceed as for the partial reduction algorithm else take for $q_{i+1}$ the pseudo-remainder of $q_{i}$ by $p$. There exists then some $\alpha \in \mathbb{N}$ such that $i_{p}^{\alpha} q_{i}=q_{i+1} \bmod (p)$. Take $h_{i+1}=i_{p}^{\alpha} h$.

\section{Properties of Ritt's reduction}

We have $q \in[A]: H_{A}^{\infty}$ if and only if ( $q$ full-rem $\left.A\right) \in[A]: H_{A}^{\infty}$. In particular, $q$ full-rem $A=0 \Rightarrow q \in[A]: H_{A}^{\infty}$. We have $q \in[A]: S_{A}^{\infty}$ if and only if $(q$ partial-rem $A) \in[A]: S_{A}^{\infty}$.

\section{Regular differential systems}

All the definitions given in this section are new (e.g. the definitions of "pairs" and "solved pairs"). We define the coherence as a property of systems of differential polynomial equations and inequations (condition C3 of definition 22) instead of the traditional property of systems of differential polynomials. This important change turns out to be very convenient and permits us to formulate Rosenfeld's Lemma for regular systems instead of coherent autoreduced sets. Though this lemma only needs $\Delta$-polynomials to be defined between elements of differentially triangular sets, we give a more general definition because we want to prove an analogue of Buchberger's second criterion in non triangular situations.

DeFInITION 17. - (critical pairs)

$A$ set $\left\{p_{1}, p_{2}\right\}$ of differential polynomials is said to be a critical pair if the leaders of $p_{1}$ and $p_{2}$ have common derivatives. If $A$ is a set of differential polynomials then critical-pairs $(A)$ denotes the set of all the pairs which can be formed between any two elements of $A$.

We do not distinguish a critical pair $\left\{p_{1}, p_{2}\right\}$ from the critical pair $\left\{p_{2}, p_{1}\right\}$. Let $\left\{p_{1}, p_{2}\right\}$ be a critical pair. It may happen that the leader of (say) $p_{2}$ is a (non necessarily proper) derivative of the leader of $p_{1}$. In that case, the critical pair $\left\{p_{1}, p_{2}\right\}$ is called a reduction critical pair. Note however we will never consider a critical pair $\left\{p_{1}, p_{2}\right\}$ such that $\operatorname{rank} p_{1}=\operatorname{rank} p_{2}$.

DeFinition 18. - ( $\Delta$-polynomials $)$

Let $\left\{p_{1}, p_{2}\right\}$ be a critical pair. Assume $\operatorname{rank} p_{1}<\operatorname{rank} p_{2}$. Denote $\theta_{1} u=$ $\operatorname{ld} p_{1}, \theta_{2} u=\operatorname{ld} p_{2}$ and $\theta_{12} u=\operatorname{lcd}\left(\theta_{1} u, \theta_{2} u\right)$. The $\Delta$-polynomial $\Delta\left(p_{1}, p_{2}\right)$ between $p_{1}$ and $p_{2}$ is defined as follows. If $\left\{p_{1}, p_{2}\right\}$ is a reduction critical pair then

$$
\Delta\left(p_{1}, p_{2}\right)=p_{2} \text { full-rem } \frac{\theta_{2}}{\theta_{1}} p_{1}
$$


else

$$
\Delta\left(p_{1}, p_{2}\right)=s_{1} \frac{\theta_{12}}{\theta_{2}} p_{2}-s_{2} \frac{\theta_{12}}{\theta_{1}} p_{1} .
$$

If $D$ is a set of critical pairs then $\Delta(D)$ denotes the set of all the $\Delta$-polynomials of its elements.

With the same notations, if $\theta_{1} u<\theta_{2} u$ then $\operatorname{ld} \Delta\left(p_{1}, p_{2}\right)<\theta_{12} u$ and there exist some $\alpha \in \mathbb{N}$ and a differential polynomial $q \in R$ such that

$$
\Delta\left(p_{1}, p_{2}\right)=s_{1}^{\alpha} \frac{\theta_{12}}{\theta_{2}} p_{2}-q \frac{\theta_{12}}{\theta_{1}} p_{1} .
$$

The notation $\Delta$ for $\Delta$-polynomials comes from Rosenfeld's paper ${ }^{4}$. Seidenberg, Rosenfeld and Kolchin never considered reduction critical pairs. Our definition coincides with theirs in the other case.

\subsection{Solved critical pairs}

DEFINITION 19. - (solved critical pairs)

A critical pair $\left\{p_{1}, p_{2}\right\}$ is said to be solved by a differential system of equations and inequations $A=0, S \neq 0$ if there exists a derivative $v<$ $\operatorname{lcd}\left(\operatorname{ld} p_{1}, \operatorname{ld} p_{2}\right)$ such that

$$
\Delta\left(p_{1}, p_{2}\right) \in\left(A_{v}\right):\left(S \cap R_{v}\right)^{\infty} .
$$

In our algorithm, we shall apply the following criterion to test whether a critical pair is solved by a differential system.

Lemma 20. - Let $\left\{p_{1}, p_{2}\right\}$ be a critical pair s.t. $\operatorname{ld} p_{1} \neq \operatorname{ld} p_{2}$. Consider a differential system $A=0, S \neq 0$ s.t. $H_{A} \subset S$. If $\Delta\left(p_{1}, p_{2}\right)$ full-rem $A=0$ then the critical pair $\left\{p_{1}, p_{2}\right\}$ is solved by $A=0, S \neq 0$.

Proof. - Denote $v=\operatorname{ld} \Delta\left(p_{1}, p_{2}\right)$. Since ld $p_{1} \neq \operatorname{ld} p_{2}$ we have $v<$ $\operatorname{lcd}\left(\operatorname{ld} p_{1}, \operatorname{ld} p_{2}\right)$. Denote $\bar{A}=\left\{p \in A \mid \operatorname{rank} p \leq \operatorname{rank} \Delta\left(p_{1}, p_{2}\right)\right\}$. According to the specifications of Ritt's reduction, there exist then $h_{1}, \ldots, h_{n} \in H_{\bar{A}}$ such that, for some positive integers $\alpha_{1}, \ldots, \alpha_{n}$ we have $h_{1}^{\alpha_{1}} \cdots h_{n}^{\alpha_{n}} \Delta\left(p_{1}, p_{2}\right) \in$ $\left(\bar{A}_{v}\right)$. Since $H_{\bar{A}} \subset H_{A} \cap R_{v}$ and $\bar{A}_{v} \subset A_{v}$ we have $\Delta\left(p_{1}, p_{2}\right) \in\left(A_{v}\right):\left(S \cap R_{v}\right)^{\infty}$ and the critical pair is solved by the differential system $A=0, S \neq 0$.

The next lemma is a generalization to a non triangular situation of a lemma already proved by [Seidenberg, 1956, inside Theorem 6, page 51], [Rosenfeld, 1959, inside Lemma, page 397] and [Kolchin, 1973, chapter IV, $\S 9$, Lemma 2].

\footnotetext{
${ }^{4}$ Note the symbol $\Delta$ has a different meaning in Kolchin's text: it denotes the set of derivations.
} 
Lemma 21. - Let $p_{1}$ and $p_{2}$ be two differential polynomials whose leaders $\theta_{1} u$ and $\theta_{2} u$ have common derivatives. Denote $s_{1}$ and $s_{2}$ their separants. Let $(\Sigma)$ denote a differential system $A=0, S \neq 0$. If $(\mathbf{H 1}) \theta_{1} u$ and $\theta_{2} u$ are different, (H2) the critical pair $\left\{p_{1}, p_{2}\right\}$ is solved by $(\Sigma)$ and (H3) $s_{1}, s_{2} \in S$ then for each derivation operator $\gamma \in \Theta$, the critical pair $\left\{\gamma p_{1}, \gamma p_{2}\right\}$ is solved by $(\Sigma)$.

Proof. - Denote $\theta_{12}=\operatorname{lcm}\left(\theta_{1}, \theta_{2}\right)$ and $\Delta_{\gamma}=\Delta\left(\gamma p_{1}, \gamma p_{2}\right)$. Denote also $\theta u=\gamma \theta_{12} u=\operatorname{lcd}\left(\operatorname{ld} \gamma p_{1}, \operatorname{ld} \gamma p_{2}\right)$.

The proof is done by induction on the order of $\gamma$. If the order is zero then the lemma is satisfied because of $\mathbf{H 2}$ else, decompose $\gamma=\delta \lambda$ where $\delta$ is a mere derivation and $\phi=\lambda \theta_{12}$. Assume (induction hypothesis) that the critical pair $\left\{\lambda p_{1}, \lambda p_{2}\right\}$ is solved by $(\Sigma)$. There exists then a derivative $v<\phi u$ and a power product $h$ of elements of $S \cap R_{v}$ such that $h \Delta_{\lambda} \in\left(A_{v}\right)$. By H1 (assuming $p_{1}<p_{2}$ ) there exist some $\alpha \in \mathbb{N}$ and a differential polynomial $q$ such that $\Delta_{\lambda}=s_{1}^{\alpha}\left(\phi / \theta_{2}\right) p_{2}-q\left(\phi / \theta_{1}\right) p_{1}$.

Consider the differential polynomial $\delta\left(h \Delta_{\lambda}\right)$. The second axiom of rankings implies that it belongs to $\left(A_{\delta v}\right)$ and that $\delta v<\theta u$. Multiply it by $h$. One gets a sum $(\delta h) h \Delta_{\lambda}+h^{2} \delta \Delta_{\lambda}$ whose first term is in $\left(A_{v}\right)$ by induction hypothesis. Since $\left(A_{v}\right) \subset\left(A_{\delta v}\right)$ we conclude $h^{2} \delta \Delta_{\lambda}$ belongs to this latter ideal. Expand this polynomial

$$
\begin{aligned}
h^{2}\left(\delta \Delta_{\lambda}\right) & =h^{2} \delta\left\{s_{1}^{\alpha} \frac{\phi}{\theta_{2}} p_{2}-q \frac{\phi}{\theta_{1}} p_{1}\right\} \\
& =h^{2}\left\{\left(\delta\left(s_{1}^{\alpha}\right)\right) \frac{\phi}{\theta_{2}} p_{2}-(\delta q) \frac{\phi}{\theta_{1}} p_{1}\right\} \\
& +h^{2}\left\{s_{1}^{\alpha} \frac{\theta}{\theta_{2}} p_{2}-q \frac{\theta}{\theta_{1}} p_{1}\right\} .
\end{aligned}
$$

The polynomials $\left(\phi / \theta_{i}\right) p_{i}(i=1,2)$ have both $\phi u<\theta u$ for leaders. If $w=$ $\max (\phi u, \delta v)$ then $w<\theta u$ and the term (3) is in $\left(A_{w}\right)$. Thus so is the term (4). Since $\theta / \theta_{2}$ and $\theta / \theta_{1}$ are proper derivation operators, we have $\Delta_{\gamma}=$ $s_{1}\left(\theta / \theta_{2}\right) p_{2}-s_{2}\left(\theta / \theta_{1}\right) p_{1}$. The term (4) is equal to $h^{2} s_{1}^{\alpha-1} \Delta_{\gamma}+C\left(\theta / \theta_{1}\right) p_{1}$ where $C$ is a differential polynomial. Using $\mathbf{H 3}$, the fact that $R_{v} \subset R_{w}$ and ld $s_{1} \leq \operatorname{ld} p_{1} \leq \phi u \leq w$, for some power product $h^{\prime}$ of elements of $S \cap R_{w}$ we have $h^{\prime} \Delta_{\gamma} \in\left(A_{w},\left(\theta / \theta_{1}\right) p_{1}\right)$. The differential polynomial $h^{\prime} \Delta_{\gamma}$ and the elements of $A_{w}$ are free of $\theta u$. Lemma 16 applies, $\Delta_{\gamma} \in\left(A_{w}\right):\left(S \cap R_{w}\right)^{\infty}$ and the critical pair $\left\{\gamma p_{1}, \gamma p_{2}\right\}$ is solved by $(\Sigma)$. 


\subsection{Rosenfeld's Lemma}

DEFINITION 22. - (regular differential systems)

$A$ differential system $A=0, S \neq 0$ of a differential polynomial ring $R$ is said to be a regular differential system (for a ranking $\mathscr{R}$ ) if

C1 $A$ is differentially triangular,

C2 $S$ contains the separants of the elements of $A$ and is partially reduced w.r.t. A,

C3 all the $\left\{p, p^{\prime}\right\} \in$ critical-pairs $(A)$ are solved by $A=0, S \neq 0$ (coherence property).

The differential ideal $[A]: S^{\infty}$ is called the regular differential ideal defined by the system.

The following lemma is a generalization of [Rosenfeld, 1959, Lemma, page 397]. which was already proved by Boulier [1997]. The first version is due to [Seidenberg, 1956, Theorem 6, page 51]. Another version was proved in [Kolchin, 1973, chapter III, §8, Lemma 5] but the part of Kolchin's lemma which is not in Rosenfeld's is not proved algorithmic. Kolchin's proof consists in a transfinite induction [van der Waerden, 1966, chapter 9]. We apply the idea in the proof of Theorem 23.

TheOREM 23. - (Rosenfeld's Lemma)

If $A=0, S \neq 0$ is a regular differential system of a differential polynomial ring $R$ for a ranking $\mathscr{R}$ then every differential polynomial in $[A]: S^{\infty}$ which is partially reduced w.r.t. $A$ belongs to $(A): S^{\infty}$.

Proof. - Let $A=\left\{p_{1}, \ldots, p_{n}\right\}$. Let $q \in[A]: S^{\infty}$ be a differential polynomial partially reduced w.r.t. $A$. Denote $F(q)$ the set of all the formulæ $(f)$ such that, for some power product $h$ of elements of $S$ we have a finite sum

$$
h q=\underbrace{\sum_{\phi \in \Theta} \sum_{j=1}^{n} B_{j, \phi} \phi p_{j}}_{(f)} .
$$

Assume $q \notin(A): S^{\infty}$. In each formula $(f) \in F(q)$ appears therefore some (at least one) proper derivatives of some leaders of elements of $A$. Denote $v(f)$ the greatest of them according to the ranking $\mathscr{R}$. Among all the formulæ $(f) \in F(q)$ let us consider one such that $v(f)$ is minimal w.r.t. $\mathscr{R}$. Such a formula exists for all rankings are well-orderings. We claim there exists another formula $\left(f^{\prime}\right) \in F(q)$ such that $v\left(f^{\prime}\right)<v(f)$. This contradiction will prove the theorem. 
Computing representations for radicals of finitely generated differential ideals 21

By Lemma 1 and the minimality hypothesis, $v(f)$ is the derivative of the leader of at least one element of $A$. Let $v(f)=\theta u$ be a proper derivative of the leaders $\theta_{1} u, \ldots, \theta_{i} u$ of the differential polynomials $p_{1}, \ldots, p_{i} \in A$, renaming the $p$ 's if needed. Denote $\left(\theta / \theta_{i}\right) p_{i}=s_{i} \theta u+r$. Apply on the terms of the formula $(f)$ the substitution $v(f) \rightarrow\left(\left(\theta / \theta_{i}\right) p_{i}-r\right) / s_{i}$ (as in Lemma 16) and multiply by some power $s_{i}^{\alpha}$ to erase denominators. Denoting $\gamma_{j}=\left(\theta / \operatorname{lcm}\left(\theta_{i}, \theta_{j}\right)\right)$ we get a formula

$$
\begin{aligned}
s_{i}^{\alpha} h q & =D \frac{\theta}{\theta_{i}} p_{i} \\
& +\sum_{j=1}^{i-1} E_{j} \Delta\left(\gamma_{j} p_{i}, \gamma_{j} p_{j}\right) \\
& +\sum_{\phi \in \Theta} \sum_{j=1}^{n} C_{j, \phi} \phi p_{j}
\end{aligned}
$$

such that only derivatives less than $v(f)$ appear in the terms of the sums (6) and (7). Since the elements of $S$ and $q$ are partially reduced w.r.t. $A$, the derivative $v(f)$ only appears in the differential polynomial $\left(\theta / \theta_{i}\right) p_{i}$. Therefore $D=0$.

If $A$ is a system of ODE the sum (6) is empty and there exists a derivative $w<v(f)$ such that $q \in\left(A_{w}\right): S^{\infty}$. Contradiction.

Assume $A=0, S \neq 0$ is a PDE system. Since it is regular, condition C3 of definition 22 holds and Lemma 21 applies: all the critical pairs $\left\{\gamma_{j} p_{i}, \gamma_{j} p_{j}\right\}$ are solved. There exists thus a derivative $w<v(f)$ such that $q \in\left(A_{w}\right): S^{\infty}$. Contradiction.

Corollary 24. - If $A=0, S \neq 0$ is a regular differential system of a differential polynomial ring $R$ then

1. we have $[A]: S^{\infty}=R$ if and only if $(A): S^{\infty}=R$,

2. for any $p \in R$ we have $p \in[A]: S^{\infty}$ iff $(p$ partial-rem $A) \in(A): S^{\infty}$,

3. a differential polynomial $p \in R$ is a divisor of zero modulo $[A]: S^{\infty}$ if and only if ( $p$ partial-rem $A$ ) is a divisor of zero modulo $(A): S^{\infty}$.

Proof. - 1. - By Rosenfeld's Lemma, $1 \in[A]: S^{\infty}$ iff $1 \in(A): S^{\infty}$. 2. - Let $p \in R$ be a differential polynomial. This point relies on the two following facts: because of condition C2, $p \in[A]: S^{\infty}$ if and only if $(p$ partial-rem $A) \in[A]: S^{\infty}$; the differential polynomial ( $p$ partial-rem $A$ ) is partially reduced w.r.t. $A$.

3. - Let $p, q \in R$ be differential polynomials. Denote $\bar{p}=p$ partial-rem $A$ 
and $\bar{q}=q$ partial-rem $A$. According to Point 2, we have $p \in[A]: S^{\infty}$ iff $\bar{p} \in(A): S^{\infty}$ and $q \in[A]: S^{\infty}$ iff $\bar{q} \in(A): S^{\infty}$. We also have $p q \in[A]: S^{\infty}$ iff $\bar{p} \bar{q} \in(A): S^{\infty}$. Therefore, $p q \in[A]: S^{\infty}$ and $p, q \notin[A]: S^{\infty}$ (i.e. $p$ is a divisor of zero modulo $[A]: S^{\infty}$ ) iff $\bar{p} \bar{q} \in(A): S^{\infty}, \bar{p}, \bar{q} \notin(A): S^{\infty}$ (i.e. $\bar{p}$ is a divisor of zero modulo $\left.(A): S^{\infty}\right)$.

THEOREM 25. - (lifting of Lazard's Lemma)

If $A=0, S \neq 0$ is a consistent regular differential system of a differential polynomial ring $R$ and $R_{0} \subset R$ denotes the ring of the differential polynomials partially reduced w.r.t. $A$ then

1. the regular differential ideal $[A]: S^{\infty}$ is radical,

2. there is a bijection between the minimal differential prime components $\mathfrak{p}_{1}, \ldots, \mathfrak{p}_{n}$ of $[A]: S^{\infty}$ and the minimal prime components $\mathfrak{b}_{1}, \ldots, \mathfrak{b}_{n}$ of $(A): S^{\infty}$ given by $\mathfrak{b}_{i}=\left(\mathfrak{p}_{i} \cap R_{0}\right)$; moreover, if $C_{i}$ is a characteristic set of $\mathfrak{b}_{i}$ then $C_{i}$ is also a characteristic set of $\mathfrak{p}_{i}$ and $\mathfrak{p}_{i}=\left[C_{i}\right]: H_{C_{i}}^{\infty}$.

Proof. - Assume $p^{k} \in[A]: S^{\infty}$ for some $k \in \mathbb{N}$. Denote $\bar{p}=$ ( $p$ partial-rem $A$ ). By Rosenfeld's Lemma $\bar{p}^{k} \in(A): S^{\infty}$. By Lazard's Lemma $\bar{p} \in(A): S^{\infty}$. By Point 2 of Corollary $24, p \in[A]: S^{\infty}$ thus $[A]: S^{\infty}$ is radical.

The ideals $\mathfrak{b}$ 's are prime and their intersection is equal to $(A): S^{\infty}$. Let's assume $(\mathbf{H} 1)$ that $\mathfrak{b}_{1}$ is redundant w.r.t. $(A): S^{\infty}$ and seek a contradiction. Let $f \in \mathfrak{p}_{2} \cap \cdots \cap \mathfrak{p}_{n}$ be a differential polynomial and $g=f$ partial-rem $A$. Since $A \subset \mathfrak{p}_{i}$ we have $g \in \mathfrak{p}_{i}$ for every $2 \leq i \leq n$. Since $g \in R_{0}$ we have $g \in \mathfrak{b}_{2} \cap \cdots \cap \mathfrak{b}_{n}$. Using $\mathbf{H 1}$ we conclude $g \in(A): S^{\infty}$. Let's summarize: ( $f$ partial-rem $A) \in(A): S^{\infty}$. By Point 2 of Corollary $24, f \in[A]: S^{\infty}$ thus $\mathfrak{p}_{1}$ is redundant w.r.t. $[A]: S^{\infty}$. Contradiction.

Assume $C_{i}$ is a characteristic set of $\left(\mathfrak{p}_{i} \cap R_{0}\right)$. Let $p \in \mathfrak{p}_{i}$ and $q=$ $p$ full-rem $C_{i}$. We have $q \in \mathfrak{p}_{i}$. By Lazard's Lemma, ld $A=\operatorname{ld} C_{i}$ thus $q \in R_{0}$. Since $q \in \mathfrak{p}_{i} \cap R_{0}$ is reduced w.r.t. $C_{i}$ we have $q=0$. Therefore $C_{i}$ is a characteristic set of $\mathfrak{p}_{i}$ and $\mathfrak{p}_{i}=\left[C_{i}\right]: H_{C_{i}}^{\infty}$.

If $A=0, S \neq 0$ is a regular differential system then the set of leaders of the elements of $A$ is equal to the set of leaders of each of the differential prime ideals which are minimal over $[A]: S^{\infty}$. All these differential prime ideals have therefore the same differential Hilbert's function. The computation of this function is then a purely combinatorial problem [Kolchin, 1973, chapter II, $\S 12]$. Moreover, by applying a primary decomposition algorithm over $(A): S^{\infty}$ we get the differential prime decomposition of the differential ideal $[A]: S^{\infty}$. Characteristic sets for the minimal differential prime components of $[A]$ : $S^{\infty}$ can then be computed using the method given by [Boulier et al., 1995, Theorem 6, page 164]. 


\subsection{Testing the coherence}

Let $A=0, S \neq 0$ be a differential system of $R$ which satisfies conditions C1 and C2 of definition 22. If $A$ is differentially triangular, $H_{A} \subset S$ and $\Delta\left(p, p^{\prime}\right)$ full-rem $A=0$ for all critical pairs $\left\{p, p^{\prime}\right\} \in \operatorname{critical}-$ pairs $(A)$ then the differential system $A=0, S \neq 0$ is regular (Lemma 20).

This criterion is useful for practical purposes but only gives a sufficient condition. Consider the next differential system $A=0, S_{A} \neq 0$ for any elimination ranking such that $u>v$. It generates only one $\Delta$-polynomial $\Delta\left(p_{1}, p_{2}\right)=v_{y}$. Now, $\Delta\left(p_{1}, p_{2}\right)\left(v_{y}+1\right)^{2} \in\left(A_{\alpha}\right)$ for some derivative $\alpha<u_{x y}$. Since $\left(v_{y}+1\right)$ is a multiple factor of $p_{3}$, it is also a factor of the separant of $p_{3}$ whence $\Delta\left(p_{1}, p_{2}\right) \in\left(A_{\alpha}\right):\left(S \cap R_{\alpha}\right)^{\infty}$. Therefore $A=0, S_{A} \neq 0$ is a regular differential system. However, the $\Delta$-polynomial $v_{y}$ is reduced w.r.t. $A$.

$$
A\left\{\begin{array}{l}
p_{1}=u_{x}+v, \\
p_{2}=u_{y}, \\
p_{3}=v_{y}\left(v_{y}+1\right)^{2} .
\end{array}\right.
$$

The following example (borrowed from [Boulier, 1997]) shows that the coherence property is only a sufficient condition ${ }^{5}$ for Rosenfeld's Lemma. Consider the following system $A$ of $\mathbb{Q}\{t, u, v, w\}$ endowed with derivations w.r.t. $x$ and $y$, for any ranking such that $t_{x}, u_{x}, u_{y}$ and $v_{y}$ are the leaders of $p_{1}, p_{2}, p_{3}$ and $p_{4}$ respectively. It generates only one critical pair $\left\{p_{2}, p_{3}\right\}$. The associated $\Delta$-polynomial is $\Delta\left(p_{2}, p_{3}\right)=v_{y}-w_{x}$.

$$
A\left\{\begin{array}{l}
p_{1}=t_{x}^{2}+v_{y} \\
p_{2}=u_{x}+v \\
p_{3}=u_{y}+w \\
p_{4}=\left(v_{y}-w_{x}\right) v_{y} .
\end{array}\right.
$$

If the ranking is assumed to be orderly, then there exists a derivative $\alpha$ such that $t_{x}, u_{x}, u_{y}, v_{y} \leq \alpha<u_{x y}$. Then $A \subset A_{\alpha}$ and $t_{x} \in S_{A} \cap R_{\alpha}$. Using $p_{1}$ and $p_{4}$ it is clear that $\Delta\left(p_{2}, p_{3}\right) t_{x}^{2} \in\left(A_{\alpha}\right)$. Since $t_{x} \in S_{A} \cap R_{\alpha}$ it follows that $\Delta\left(p_{2}, p_{3}\right) \in\left(A_{\alpha}\right):\left(S_{A} \cap R_{\alpha}\right)^{\infty}$ i.e. the critical pair $\left\{p_{2}, p_{3}\right\}$ is solved by $A=0, S \neq 0$. This differential system is thus regular and Rosenfeld's Lemma applies. If the ranking is an elimination ranking such that $t>u$ then for each derivative $\alpha<u_{x y}$ we have $p_{1} \notin A_{\alpha}$ and $t_{x} \notin S_{A} \cap R_{\alpha}$. It can be proved [Boulier, 1997, Lemma 6] that $\Delta\left(p_{2}, p_{3}\right) \notin\left(A_{\alpha}\right):\left(S \cap R_{\alpha}\right)^{\infty}$ i.e. the critical pair $\left\{p_{2}, p_{3}\right\}$ is not solved by $A=0, S \neq 0$. This differential system is not regular w.r.t. this latter ranking. However, since the leaders and the

\footnotetext{
${ }^{5}$ Our condition of coherence, which is based on solved pairs, is not equivalent to that of Rosenfeld. Our example applies to our definition, not to that of Rosenfeld.
} 
families $S_{A}$ are the same for both rankings, the conclusion of Rosenfeld's Lemma still holds.

\subsubsection{Buchberger's criteria}

Most of the results of this section are borrowed from [Boulier, 1997]. Buchberger [1979] established a few criteria which predict that some $S$-polynomials [Becker and Weispfenning, 1991, Definition 5.46] are reduced to zero without having to actually reduce them. They turn out to be very important in practice since most of the CPU time is spent in $S$-polynomials reductions. Remark however they do not change the theoretical complexity of Gröbner bases since this complexity expresses the size of the Gröbner basis (which does not depend on the algorithm) in terms of the size of the input system.

Buchberger's first criterion [Becker and Weispfenning, 1991, Lemma 5.66] states that if the leading terms of two polynomials $p$ and $q$ are disjoint (i.e. their least common multiple is equal to their product) then the $S$-polynomial $S(p, q)$ gets reduced to zero by the set $\{p, q\}$. In differential algebra, we might conjecture that if $p$ and $q$ are two differential polynomials with leaders $\theta u$ and $\phi u$ respectively and if $\theta$ and $\phi$ are disjoint then the $\Delta$-polynomial $\Delta(p, q)$ full-rem $\{p, q\}=0$. This conjecture is false in general but true in the next case.

Proposition 26. - (analogue of Buchberger's first criterion)

If $p$ and $q$ are two differential polynomials which are linear, homogeneous, in one differential indeterminate, with constant coefficients and if (denoting $\operatorname{ld} p=\theta u$ and $\operatorname{ld} q=\phi u)$ we have $\operatorname{lcd}(\theta u, \phi u)=\theta \phi u$ then $\Delta(p, q)$ full-rem $\{p, q\}=0$.

Proof. - Let $R=K\{u\}$ be a differential polynomial ring endowed with a ranking and a set of derivations $\left\{\delta_{1}, \ldots, \delta_{m}\right\}$. Let $\bar{R}=K\left[x_{1}, \ldots, x_{m}\right]$ be a non differential polynomial ring. To each differential polynomial $f=\theta_{1} u+$ $\cdots+\theta_{s} u$ which is linear, homogeneous and with constant coefficients we may associate a polynomial $\gamma f \in \bar{R}$ defined by $\gamma f=\gamma \theta_{1} u+\cdots+\gamma \theta_{s} u, \gamma c=c$ for every $c \in K$ and $\gamma\left(\delta_{1}^{\alpha_{1}} \cdots \delta_{m}^{\alpha_{m}} u\right)=x_{1}^{\alpha_{1}} \cdots x_{m}^{\alpha_{m}}$. The monoid of terms over the alphabet $\left\{x_{1}, \ldots, x_{m}\right\}$ is endowed with the admissible ordering [Becker and Weispfenning, 1991, Definition 4.59] given by the ranking. Let $p, q, r \in R$ satisfying the hypotheses of the proposition. On the one hand, $\Delta(p, q)=$ $\gamma^{-1} S(\gamma p, \gamma q)$; on the other hand $r$ full-rem $\{p, q\}=\bar{r}$ iff $\gamma r$ gets reduced to $\gamma \bar{r}$ by the set $\{\gamma p, \gamma q\}$. By Buchberger's first criterion, $S(\gamma p, \gamma q)$ is reduced to zero by $\{\gamma p, \gamma q\}$ thus $\Delta(p, q)$ full-rem $\{p, q\}=0$.

The following example shows that the conjecture is false if the equations are not homogeneous: take $p=u_{x}+1$ and $q=u_{y}+u$. The $\Delta$-polynomial 
$\Delta(p, q)=u_{x}$ is reduced to 1 by the set $\{p, q\}$.

This one shows that the conjecture is false if the coefficients of the equations are not constants: assume the coefficient $c$ is such that $c_{y}=1$ and take $p=u_{x}+c u$ and $q=u_{y}$. The $\Delta$-polynomial $\Delta(p, q)=c u_{y}+u$ is reduced to $u$ by $\{p, q\}$.

In Proposition 27, we prove an analogue of Buchberger's second criterion. However we impose restrictions on the differential polynomials which have no counterpart in the Gröbner bases theory. This makes the proof of its implementation in the Rosenfeld-Gröbner algorithm more painful than in the non differential case.

Proposition 27. - (analogue of Buchberger's second criterion)

Let $\left\langle p_{1}, p_{2}, p_{3}\right\rangle$ be a triple of differential polynomials such that $(\mathbf{H} 1)$ the leaders $\theta_{1} u, \theta_{2} u$ and $\theta_{3} u$ of the $p$ 's have common derivatives and are pairwise different, (H2) $\operatorname{lcd}\left(\theta_{1} u, \theta_{3} u\right)$ is a derivative of $\theta_{2} u$ and $\mathbf{( H 3 )}$ one of the following conditions holds:

1. $\operatorname{ld} p_{i}$ is not a derivative of $\operatorname{ld} p_{j}(1 \leq i, j \leq 3$ and $i \neq j)$,

2. $p_{1}<p_{2}<p_{3}$ or $p_{3}<p_{2}<p_{1}$,

3. $p_{2}<p_{1}<p_{3}$ and $\operatorname{deg}\left(p_{1}, \theta_{1} u\right)=1$,

4. $p_{1}<p_{3}<p_{2}$ and $\operatorname{deg}\left(p_{3}, \theta_{3} u\right)=1$.

Let $A=0, S \neq 0$ be a differential system. If $(\mathbf{H} 4)$ the critical pairs $\left\{p_{1}, p_{2}\right\}$ and $\left\{p_{2}, p_{3}\right\}$ are solved by $A=0, S \neq 0$ and (H5) $s_{1}, s_{2}, s_{3} \in S$ then the critical pair $\left\{p_{1}, p_{3}\right\}$ is solved by $A=0, S \neq 0$.

Proof. - Denote $\theta_{i j} u=\operatorname{lcd}\left(\theta_{i} u, \theta_{j} u\right)$. Because of $\mathbf{H 2}$ the derivation operators $\left(\theta_{13} / \theta_{12}\right)$ and $\left(\theta_{13} / \theta_{23}\right)$ exist. Denote

$$
\begin{aligned}
& \Delta_{3}=\Delta\left(\frac{\theta_{13}}{\theta_{12}} p_{1}, \frac{\theta_{13}}{\theta_{12}} p_{2}\right), \\
& \Delta_{1}=\Delta\left(\frac{\theta_{13}}{\theta_{23}} p_{2}, \frac{\theta_{13}}{\theta_{23}} p_{3}\right) .
\end{aligned}
$$

Lemma: if there exist differential polynomials $B, C$ and $D$ and a power product $h$ of elements of $S$ such that ld $h<\theta_{13} u$ and

$$
h \Delta\left(p_{1}, p_{3}\right)=B \Delta_{3}+C \Delta_{1}+D \frac{\theta_{13}}{\theta_{1}} p_{1}
$$

then the critical pair $\left\{p_{1}, p_{3}\right\}$ is solved by $A=0, S \neq 0$. Proof: by $\mathbf{H} \mathbf{4}$ and Lemma 21 there exists a derivative $v<\theta_{13} u$ such that $\Delta_{3}, \Delta_{1} \in\left(A_{v}\right):(S \cap$ 
$\left.R_{v}\right)^{\infty}$. By $\mathbf{H} 5$ there exists a power product $h^{\prime}$ of elements of $S$ such that $h^{\prime} \Delta\left(p_{1}, p_{3}\right) \in\left(A_{v},\left(\theta_{13} / \theta_{1}\right) p_{1}\right)$ and $\operatorname{ld} h^{\prime}=\max (\operatorname{ld} h, v)<\theta_{13} u$. Denote $w=$ $\max \left(\theta_{1} u\right.$, ld $\left.h^{\prime}\right)$. Because of $\mathbf{H 1}$ we have $w<\theta_{13} u$. By $\mathbf{H} \mathbf{5}$ and Lemma 16, $\Delta\left(p_{1}, p_{3}\right) \in\left(A_{w}\right):\left(S \cap R_{w}\right)^{\infty}$ and the critical pair $\left\{p_{1}, p_{3}\right\}$ is solved by the system $A=0, S \neq 0$. This concludes the proof of the lemma.

If $\operatorname{ld} p_{i}$ is not a derivative of $\operatorname{ld} p_{j}(1 \leq i, j \leq 3$ and $i \neq j)$ then $s_{2} \Delta\left(p_{1}, p_{3}\right)=s_{1} \Delta_{1}+s_{3} \Delta_{3}$. Because of $\mathbf{H 1}$ we have ld $s_{2}<\theta_{13} u$. By $\mathbf{H} \mathbf{5}$ our lemma above applies and the critical pair $\left\{p_{1}, p_{3}\right\}$ is solved by $A=0, S \neq 0$. If $p_{1}<p_{2}<p_{3}$ then there exist some $\alpha_{1}, \alpha_{2}, \alpha_{3} \in \mathbb{N}$ and some differential polynomials $q_{1}, q_{2}$ and $q_{3}$ such that

$$
\begin{aligned}
\Delta_{3} & =s_{1}^{\alpha_{3}} \frac{\theta_{13}}{\theta_{2}} p_{2}-q_{3} \frac{\theta_{13}}{\theta_{1}} p_{1}, \\
\Delta_{1} & =s_{2}^{\alpha_{1}} \frac{\theta_{13}}{\theta_{3}} p_{3}-q_{1} \frac{\theta_{13}}{\theta_{2}} p_{2}, \\
\Delta\left(p_{1}, p_{3}\right) & =s_{1}^{\alpha_{2}} \frac{\theta_{13}}{\theta_{3}} p_{3}-q_{2} \frac{\theta_{13}}{\theta_{1}} p_{1} .
\end{aligned}
$$

Denoting $\beta=\max \left(\alpha_{2}, \alpha_{3}\right)$, there exists a differential polynomial $C$ such that

$$
s_{1}^{\beta-\alpha_{2}} s_{2}^{\alpha_{1}} \Delta\left(p_{1}, p_{3}\right)=s_{1}^{\beta} \Delta_{1}+q_{1} s_{1}^{\beta-\alpha_{3}} \Delta_{3}+C \frac{\theta_{13}}{\theta_{1}} p_{1} .
$$

Because of $\mathbf{H 1}$ we have $\operatorname{ld}\left(s_{1}^{\beta-\alpha_{2}} s_{2}^{\alpha_{1}}\right)<\theta_{13} u$. By $\mathbf{H} 5$ our lemma applies and the critical pair $\left\{p_{1}, p_{3}\right\}$ is solved by $A=0, S \neq 0$. If $p_{2}<p_{1}<p_{3}$ then

$$
\Delta_{3}=s_{2}^{\alpha_{3}} \frac{\theta_{13}}{\theta_{1}} p_{1}-q_{3} \frac{\theta_{13}}{\theta_{2}} p_{2}
$$

Computing as above we find a relation

$$
q_{3} s_{2}^{\alpha_{1}} \Delta\left(p_{1}, p_{3}\right)=q_{1} s_{1}^{\alpha_{2}} \Delta_{3}-q_{3} s_{1}^{\alpha_{2}} \Delta_{1}+C \frac{\theta_{13}}{\theta_{1}} p_{1} .
$$

In the general case, one cannot apply our lemma for $q_{3} \notin S$. Assume that $\operatorname{deg}\left(p_{1}, \theta_{1} u\right)=1$. By $\mathbf{H} 5$ we have $q_{3}=s_{1} \in S$. Because of $\mathbf{H 1}$, we have $\operatorname{ld}\left(q_{3} s_{2}^{\alpha_{1}}\right)<\theta_{13} u$. Our lemma applies and the critical pair $\left\{p_{1}, p_{3}\right\}$ is solved by $A=0, S \neq 0$. The last case is similar to the former one. 


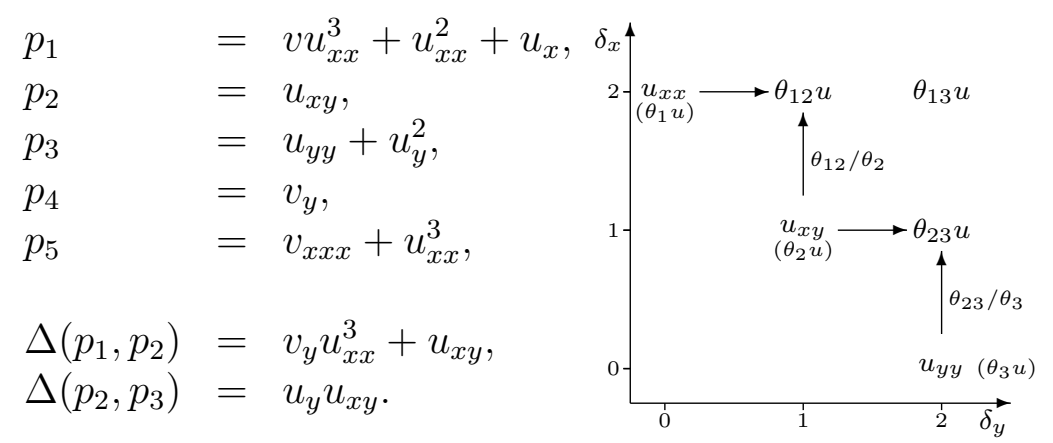

The picture illustrates Proposition 27 in the triangular case. Both $\Delta\left(p_{1}, p_{2}\right)$ and $\Delta\left(p_{2}, p_{3}\right)$ are reduced to zero by $A$. Therefore the critical pairs $\left\{p_{1}, p_{2}\right\}$ and $\left\{p_{2}, p_{3}\right\}$ are solved by the system $A=0, H_{A} \neq 0$. The least common derivative between the leaders of $p_{1}$ and $p_{3}$ is a derivative of the leader of $p_{2}$. Thus the critical pair $\left\{p_{1}, p_{3}\right\}$ is solved by the system.

\section{Computing a regular decomposition}

This section aims at proving Theorem 28 which constitutes the core of the Rosenfeld-Gröbner algorithm. Our implementation of the algorithm, given in Figure 1, can be viewed as a mere translation in some pseudo programming language of the effective proof of this theorem.

Our implementation carries the analogue of Buchberger's second criterion out. It is a lifting for the differential algebra of the version of Buchberger's algorithm by Gebauer and Möller [1988]. The book of [Becker and Weispfenning, 1991, pages 230-232] furnished us many important informations on that subject. It is much more efficient than the ones given by Boulier [1994] or Boulier et al. [1995].

THEOREM 28. - (computing a regular decomposition)

If $P_{0}=0, S_{0} \neq 0$ is a differential system of a differential polynomial ring $R$ then it is possible to compute finitely many consistent regular differential systems $A_{i}=0, S_{i} \neq 0(1 \leq i \leq n)$ such that

$$
\mathfrak{p}=\sqrt{\left[P_{0}\right]: S_{0}^{\infty}}=\left[A_{1}\right]: S_{1}^{\infty} \cap \cdots \cap\left[A_{n}\right]: S_{n}^{\infty} .
$$

This decomposition may contain components redundant w.r.t. $\mathfrak{p}$. Operations needed are addition, multiplication, differentiation and equality test with zero in the base field of $R$.

A quadruple $G=\langle A, D, P, S\rangle$ is a data structure which contains a differential system being processed until it is regular. The set $A \subset R$ contains equations already processed. The set $P \subset R$ contains the equations which 
are not yet processed. The set $D$ contains critical pairs which have to be solved and $S \subset R$ contains the inequations.

Initially, $P=P_{0}, S=S_{0}$ and $A=D=\varnothing$. If $P \neq \varnothing$ or $D \neq \varnothing$ then the current quadruple is rewritten as finitely many quadruples by a completion and splitting process. If $P=D=\varnothing$ then an autoreduction process transforms the differential system $A=0, S \neq 0$ as an equivalent regular differential system $\bar{A}=0, \bar{S} \neq 0$. The autoreduction process decides if the system is consistent or not. In the former case, the regular differential ideal $[\bar{A}]: \bar{S}^{\infty}$ becomes one of the components of intersection (9); in the latter, the system is discarded.

Let $G=\langle A, D, P, S\rangle$ be a quadruple. We denote by $\mathscr{P}(D)$ the set of all the differential polynomials $p$ such that there exists a reduction critical pair $^{6}\left\{p, p^{\prime}\right\} \in D$ with $\operatorname{rank} p>\operatorname{rank} p^{\prime}$. Let $\mathscr{F}(G)=A \cup \mathscr{P}(D) \cup P$ and $\mathscr{I}(G)=\sqrt{[\mathscr{F}(G)]: S^{\infty}}$. The solutions of a quadruple $G$ are defined as the solutions of the differential system $\mathscr{F}(G)=0, S \neq 0$. A critical pair is said to be solved by $G$ if it is solved by the system $\mathscr{F}(G)=0, S \neq 0$. The following axioms give the definition of critical pairs nearly solved by $G$.

A1 Every critical pair which is solved by $G$ is nearly solved by $G$.

A2 Every critical pair which belongs to $D$ is nearly solved by $G$.

A3 If $\left\{p_{1}, p_{2}\right\}$ and $\left\{p_{2}, p_{3}\right\}$ are critical pairs nearly solved by $G$ and if the triple $\left\langle p_{1}, p_{2}, p_{3}\right\rangle$ satisfies the hypotheses $\mathbf{H 1}, \mathbf{H} 2$ and $\mathbf{H} 3$ of Proposition 27 then the critical pair $\left\{p_{1}, p_{3}\right\}$ is nearly solved by $G$.

We are now ready to state some properties which will become loop invariants of our implementation of the Rosenfeld-Gröbner algorithm. Let $G=\langle A, D, P, S\rangle$ be a quadruple.

I1 The rank of the set $A$ is autoreduced.

I2 If $\left\{p, p^{\prime}\right\} \in D$ is a reduction critical pair with $\operatorname{rank} p>\operatorname{rank} p^{\prime}=v^{d}$ and $F=\left\{f \in \mathscr{F}(G) \mid \operatorname{rank} f \leq v^{d}\right\}$ then $p^{\prime} \in\left(F_{v}\right):\left(S \cap R_{v}\right)^{\infty}$.

I3 Every critical pair $\left\{p, p^{\prime}\right\} \in \operatorname{critical}-$ pairs $(A)$ is nearly solved by $G$.

I4 If $p \in A$ or $p$ belongs to some critical pair of $D$ then $i_{p}, s_{p} \in S$.

I5 If $\left\{p, p^{\prime}\right\} \in D$ is not a reduction critical pair then $\Delta\left(p, p^{\prime}\right) \in \mathscr{I}(G)$.

I6 If $\left\{p, p^{\prime}\right\} \in D$ is a critical pair then $\operatorname{rank} p \neq \operatorname{rank} p^{\prime}$.

\footnotetext{
${ }^{6}$ Recall we don't distinguish $\left\{p, p^{\prime}\right\}$ from $\left\{p^{\prime}, p\right\}$.
} 


\subsection{The final autoreduction process}

Let $G=\langle A, D, P, S\rangle$ be a quadruple satisfying the invariants and s.t. $D=$ $P=\varnothing$. The differential system is not necessarily regular. We present here one possible way to transform it as an equivalent regular differential system. This process may show that $\mathscr{I}(G)=R$. In that case, the quadruple $G$ is discarded. We build a sequence of differential systems. Let

$$
A_{0}=A, \quad S_{0}=S
$$

Let $k \geq 0$ be an index. If $A_{k}$ is not differentially triangular then let $\theta u$ be the greatest derivative occuring in some $p \in A_{k}$ being also a proper derivative of the leader $\theta^{\prime} u$ of some $p^{\prime} \in A_{k}$. Denoting $\phi=\theta / \theta^{\prime}$, compute $\bar{p}=p$ full-rem $\phi p^{\prime}$ and

$$
\begin{aligned}
A_{k+1} & =A_{k} \backslash\{p\} \cup\{\bar{p}\}, \\
S_{k+1} & =S_{k} \cup\left\{i_{\bar{p}}, s_{\bar{p}}\right\} .
\end{aligned}
$$

If $\operatorname{rank} A_{k} \neq \operatorname{rank} A_{k+1}$ then $\mathscr{I}(G)=R$ (proved below) and the quadruple is discarded. Let's assume $\operatorname{rank} A_{k}=\operatorname{rank} A_{k+1}$. If $A_{k}$ is differentially triangular then take

$$
\bar{A}=A_{k}, \quad \bar{S}=S_{k} \text { partial-rem } \bar{A} .
$$

Proposition 29. - The autoreduction process terminates.

ProOF. - The sequence of the rewritten derivatives $\theta u$ is strictly decreasing and rankings are well orderings.

Proposition 30. - For each $k \geq 0$ we have $H_{A_{k}} \subset S_{k}$. Moreover, $H_{\bar{A}} \subset$ $\bar{S}$.

Proof. - The first statement is clear. The second one is due to the fact that, since $\bar{A}$ is differentially triangular, the initials and the separants of its elements are partially reduced w.r.t. it, and are thus left inchanged by the final partial reduction.

Proposition 31. - For each $k \geq 0$, if $\operatorname{rank} A_{k} \neq \operatorname{rank} A_{k+1}$ then $\left[A_{k}\right]: S_{k}^{\infty}=$ $R$.

Proof. - If rank $A_{k} \neq \operatorname{rank} A_{k+1}$ then some initial $i_{p}$ of some element of $A_{k}$ has been reduced to zero. By Proposition 30 we have $i_{p} \in\left[A_{k}\right]: S_{k}^{\infty}$. Since $i_{p} \in S_{k}$ (by Proposition 30 again) $\left[A_{k}\right]: S_{k}^{\infty}=R$.

Let us now explicit the values of $\bar{p}$, its initial and its separant. Let $k \geq 0$ be an index. Since rank $A_{k}$ is autoreduced, $\operatorname{ld} \phi p^{\prime}=\theta u<\operatorname{ld} p$ and there exist 
some $\alpha \in \mathbb{N}$ and differential polynomials $q_{0}, q_{1}, q_{2} \in R_{\theta u}$ such that

$$
\begin{aligned}
\bar{p} & =s_{p^{\prime}}^{\alpha} p-q_{0} \phi p^{\prime}, \\
s_{\bar{p}} & =s_{p^{\prime}}^{\alpha} s_{p}-q_{1} \phi p^{\prime}, \\
i_{\bar{p}} & =s_{p^{\prime}}^{\alpha} i_{p}-q_{2} \phi p^{\prime} .
\end{aligned}
$$

Lemma 32. - If $\operatorname{rank} A_{k}=\operatorname{rank} A_{k+1}$ then for every derivative $v$ we have

$$
\left(A_{k, v}\right):\left(S_{k} \cap R_{v}\right)^{\infty} \subset\left(A_{k+1, v}\right):\left(S_{k+1} \cap R_{v}\right)^{\infty} .
$$

Proof. - First observe $S_{k} \subset S_{k+1}$. If $v<\operatorname{ld} p$ then $A_{k, v}=A_{k+1, v}$. If $v \geq \operatorname{ld} p$, it suffices to prove $p \in\left(A_{k+1, v}\right):\left(S_{k+1} \cap R_{v}\right)^{\infty}$. Since $\operatorname{ld} p=\operatorname{ld} \bar{p}$ we have $\bar{p} \in A_{k+1, v}$. Since ld $\phi p^{\prime}=\theta u<\operatorname{ld} p$ we have $\phi p^{\prime} \in A_{k+1, v}$ and $s_{p^{\prime}} \in R_{v}$. Using (10) and Proposition 30, we conclude $p \in\left(A_{k+1, v}\right):\left(S_{k+1} \cap R_{v}\right)^{\infty}$.

Proposition 33. - For each index $k \geq 0$, we have $\left[A_{k}\right]: S_{k}^{\infty}=\left[A_{k+1}\right]: S_{k+1}^{\infty}$.

Proof. - The inclusion $\left[A_{k}\right]: S_{k}^{\infty} \subset\left[A_{k+1}\right]: S_{k+1}^{\infty}$ comes from Lemma 32. The converse one. Assume $f$ is a differential polynomial such that, for some $\beta, \gamma \in \mathbb{N}$ we have $i_{\bar{p}}^{\beta} s_{\bar{p}}^{\gamma} f \in\left[A_{k+1}\right]$. By relation (10) we have $\bar{p} \in\left[A_{k}\right]: S_{k}^{\infty}$ hence $\left[A_{k+1}\right] \subset\left[A_{k}\right]: S_{k}^{\infty}$. By relations (11) and (12) and the fact that $s_{p}, s_{p^{\prime}}, i_{p} \in S_{k}$ (Proposition 30) $f \in\left[A_{k}\right]: S_{k}^{\infty}$.

Lemma 34. - Assume that all the sets $A_{k}$ have the same rank. Then all critical pairs in critical-pairs $\left(A_{k}\right)$ are solved by $A_{k}=0, S_{k} \neq 0$.

Proof. - The proof is an induction on $k$. Basis of the induction. Every critical pair $\left\{p, p^{\prime}\right\} \in$ critical-pairs $\left(A_{0}\right)$ is solved by $A_{0}=0, S_{0} \neq 0$ by I3, the fact that $D=\varnothing$ and Proposition 27 .

The general case. Let $k \geq 0$ be an index. We assume (induction hypothesis) that all critical pairs in critical-pairs $\left(A_{k}\right)$ are solved by $A_{k}=0, S_{k} \neq 0$ and we prove that, if $\left\{p_{1}, p_{2}\right\} \in$ critical-pairs $\left(A_{k+1}\right)$ then $\left\{p_{1}, p_{2}\right\}$ is solved by $A_{k+1}=0, S_{k+1} \neq 0$.

First subcase: $p_{1} \neq \bar{p}$ and $p_{2} \neq \bar{p}$. Then $\left\{p_{1}, p_{2}\right\} \in \operatorname{critical}-\operatorname{pairs}\left(A_{k}\right)$ is solved by the system $A_{k}=0, S_{k} \neq 0$ i.e. there exists some $v<\operatorname{lcd}\left(\operatorname{ld} p_{1}, \operatorname{ld} p_{2}\right)$ such that $\Delta\left(p_{1}, p_{2}\right) \in\left(A_{k, v}\right):\left(S_{k} \cap R_{v}\right)^{\infty}$. By Lemma $32 \Delta\left(p_{1}, p_{2}\right) \in$ $\left(A_{k+1, v}\right):\left(S_{k+1} \cap R_{v}\right)^{\infty}$ and the critical pair is solved by $A_{k+1}=0, S_{k+1} \neq 0$. Second subcase: $p_{1}=\bar{p}$. Since $\operatorname{rank} A_{k}=\operatorname{rank} A_{k+1}$ we have $\operatorname{ld} p=\operatorname{ld} \bar{p}=$ ld $p_{1}=\theta_{1} u$ and (assuming with no loss of generality that $\bar{p}<p_{2}$ )

$$
\Delta\left(\bar{p}, p_{2}\right)=s_{\bar{p}} \frac{\theta_{12}}{\theta_{2}} p_{2}-s_{p_{2}} \frac{\theta_{12}}{\theta_{1}} \bar{p} .
$$

\footnotetext{
${ }^{7}$ By $A_{k, v}$ we mean $E_{v}$ where $E=A_{k}$.
} 
Expanding the value of $\Delta\left(\bar{p}, p_{2}\right)$ using formulæ (10) and (11) and recalling ld $\phi p^{\prime}<\operatorname{ld} p_{1}$ we see there exists a derivative $v<\theta_{12} u$ such that $s_{p^{\prime}}^{\alpha} \Delta\left(p, p_{2}\right) \equiv$ $\Delta\left(\bar{p}, p_{2}\right)\left(\bmod \left(A_{k, v}\right)\right)$. By the fact that the critical pair $\left\{p, p_{2}\right\}$ is solved by $A_{k}=0, S_{k} \neq 0$ (induction hypothesis) and Lemma 32 the critical pair $\left\{\bar{p}, p_{2}\right\}$ is solved by $A_{k+1}=0, S_{k+1} \neq 0$.

Proposition 35. - Every critical pair in critical-pairs $(\bar{A})$ is solved by the system $\bar{A}=0, \bar{S} \neq 0$.

Proof. - According to Lemma 34 every critical pair in critical-pairs $(\bar{A})$ is solved by $\bar{A}=0, S_{k} \neq 0$, where $k$ is the index such that $\bar{A}=A_{k}$ is differentially triangular. It suffices to prove that for any derivative $v$ we have $\left(\bar{A}_{v}\right):\left(S_{k} \cap R_{v}\right)^{\infty} \subset\left(\bar{A}_{v}\right):\left(\bar{S} \cap R_{v}\right)^{\infty}$. Let $s \in S_{k} \cap R_{v}$ be not partially reduced w.r.t. $A$ and $\bar{s}=s$ partial-rem $A$. There exists a power product $h$ of elements of $S_{A} \cap R_{v}$ such that $h s \equiv \bar{s}\left(\bmod \left(A_{v}\right)\right)$. By Proposition 30 we have $S_{A} \subset \bar{S}$ and the proposition is proved.

By Propositions 31 and 33, if the rank of the set $A_{k}$ changes during the autoreduction process then the differential system $A=0, S \neq 0$ is proved to be inconsistent and can be discarded. Let's assume this is not the case. The system $\bar{A}=0, \bar{S} \neq 0$ is a regular differential system. Indeed $\bar{A}$ is differentially triangular (condition $\mathbf{C} 1$ is satisfied); $\bar{S}$ contains $H_{\bar{A}}$ (Proposition 30) and is partially reduced w.r.t. $\bar{A}$ (condition C2 is satisfied); Proposition 35 proves condition C3 holds for $\bar{A}=0, \bar{S} \neq 0$. Computing a Gröbner basis of the ideal $(\bar{A}): \bar{S}^{\infty}$ in dimension zero, one decides whether the regular differential system $\bar{A}=0, \bar{S} \neq 0$ is consistent (Point 1 of Corollary 24). If it is inconsistent, it is discarded. Otherwise, $\mathscr{I}(G)=[\bar{A}]: \bar{S}^{\infty}$ by Point 1 of Theorem 25.

\subsection{The completion process}

We consider a quadruple $G=\langle A, D, P, S\rangle$ satisfying the invariants and such that $D \neq \varnothing$ or $P \neq \varnothing$. Roughly speaking, we pick a new equation $q=0$ from these sets, reduce it by $A$ and enlarge $A$ with it (if non zero of course). Applying the analogues of Buchberger's criteria, we do not only try to generate as few critical pairs as possible but also to remove as many critical pairs as possible from $D$. The method is not optimal. Gebauer and Möller's version of the Buchberger algorithm is not either.

Pick either a differential polynomial $q_{0} \in P$ or a critical pair $\left\{p_{0}, p_{0}^{\prime}\right\} \in D$. In the former case, let $P^{*}=P \backslash\left\{q_{0}\right\}$ let $D^{*}=D$ and $q=q_{0}$ full-rem $A$. In the latter let $P^{*}=P$, let $D^{*}=D \backslash\left\{\left\{p_{0}, p_{0}^{\prime}\right\}\right\}$ and $q=\Delta\left(p_{0}, p_{0}^{\prime}\right)$ full-rem $A$. Assume that $q \neq 0$ and that $G^{\prime}=\left\langle A^{\prime}, D^{\prime}, P^{\prime}, S^{\prime}\right\rangle$ be the quadruple returned by the complete function given in Figure 2 . 
Lemma 36. - $A \subset A^{\prime} \cup \mathscr{P}\left(D^{\prime}\right)$.

Proof. - It suffices to show that if $p \in A$ is such that $\operatorname{ld} p$ is a derivative of ld $q$ then the reduction critical pair $\{p, q\}$ is kept ${ }^{8}$ in $D_{1}$. By the hypothesis H2 of Proposition 27, if $\{p, q\}$ is not kept in $D_{1}$, there exists a differential polynomial $p^{\prime} \in A$ such that $\operatorname{lcd}(\operatorname{ld} p, \operatorname{ld} q)=\operatorname{ld} p$ is a derivative of $\operatorname{ld} p^{\prime}$. This is impossible for $p, p^{\prime} \in A$ and $\operatorname{rank} A$ is autoreduced.

Lemma 37. - If $\left\{p, p^{\prime}\right\} \in D^{*}$ is a reduction critical pair then $\left\{p, p^{\prime}\right\} \in D_{2}$.

Proof. - Assume $\operatorname{rank} p>\operatorname{rank} p^{\prime}$. Since $\left\{p, p^{\prime}\right\}$ is a reduction critical pair we have $\operatorname{lcd}\left(\operatorname{ld} p, \operatorname{ld} p^{\prime}\right)=\operatorname{ld} p$. Thus, if the triple $\left\langle p, q, p^{\prime}\right\rangle$ satisfies the hypothesis $\mathbf{H} \mathbf{2}$ of Proposition 27 then $\operatorname{ld} p$ is a derivative of $\operatorname{ld} q$ hence $\operatorname{lcd}(\operatorname{ld} p, \operatorname{ld} q)=\operatorname{ld} p=\operatorname{lcd}\left(\operatorname{ld} p, \operatorname{ld} p^{\prime}\right)$ and the critical pair is kept in $D_{2}$.

Lemma 38. - If $v^{d}$ is any rank, $F=\left\{p \in \mathscr{F}(G) \mid \operatorname{rank} p \leq v^{d}\right\}$ and $F^{\prime}=\left\{p \in \mathscr{F}\left(G^{\prime}\right) \mid \operatorname{rank} p \leq v^{d}\right\}$ then $\left(F_{v}\right):\left(S \cap R_{v}\right)^{\infty} \subset\left(F_{v}^{\prime}\right):\left(S^{\prime} \cap R_{v}\right)^{\infty}$.

Proof. - Denote $F^{*}=\left\{p \in A \cup \mathscr{P}\left(D^{*}\right) \cup P^{*} \mid \operatorname{rank} p \leq v^{d}\right\}$. By Lemmas 36 and 37 we have $F^{*} \subset F^{\prime}$. We thus have two cases to consider. First case: $P^{*} \neq P$. Precisely, we assume $q=q_{0}$ full-rem $A$ with $q_{0} \in P$ and we prove that, if rank $q_{0} \leq v^{d}$ then $q_{0} \in\left(F_{v}^{\prime}\right):\left(S^{\prime} \cap R_{v}\right)^{\infty}$. This comes from Lemma 36, the fact that the elements of $A$ involved in the reduction process of $q_{0}$ have rank lower than or equal to that of $q_{0}$ that $H_{A} \subset S \subset S^{\prime}$ that $q \in A^{\prime}$ and $\operatorname{rank} q \leq \operatorname{rank} q_{0}$.

Second case: $\mathscr{P}\left(D^{*}\right) \neq \mathscr{P}(D)$. Precisely, we assume $q=\Delta\left(p_{0}, p_{0}^{\prime}\right)$ full-rem $A$ and $\left\{p_{0}, p_{0}^{\prime}\right\}$ is a reduction critical pair with rank $p_{0}>\operatorname{rank} p_{0}^{\prime}$. We prove that, if $\operatorname{rank} p_{0} \leq v^{d}$ then $p_{0} \in\left(F_{v}^{\prime}\right):\left(S^{\prime} \cap R_{v}\right)^{\infty}$.

Claim: there exists a power product $h$ of elements of $S^{\prime} \cap R_{v}$ such that $h p_{0} \equiv \Delta\left(p_{0}, p_{0}^{\prime}\right) \bmod \left(F_{v}^{\prime}\right)$. Since $\left\{p_{0}, p_{0}^{\prime}\right\}$ is a reduction critical pair, there exists some derivation operator $\phi$ such that $\Delta\left(p_{0}, p_{0}^{\prime}\right)=p_{0}$ full-rem $\phi p_{0}^{\prime}$. Thus there exist $\alpha, \beta \in \mathbb{N}$ such that $i_{p_{0}^{\prime}}^{\alpha} s_{p_{0}^{\prime}}^{\beta} p_{0} \equiv \Delta\left(p_{0}, p_{0}^{\prime}\right) \bmod \left(\phi p_{0}^{\prime}\right)$. Using Lemmas 36 and 37, the fact that $G$ satisfies $\mathbf{I} 2$ and $\operatorname{rank} p_{0}^{\prime}<\operatorname{rank} p_{0} \leq v^{d}$ we see $p_{0}^{\prime} \in\left(F_{v}^{\prime}\right):\left(S^{\prime} \cap R_{v}\right)^{\infty}$. Since ld $\phi p_{0}^{\prime}=\operatorname{ld} p_{0} \leq v$, we have $\phi p_{0}^{\prime} \in$ $\left(F_{v}^{\prime}\right):\left(S^{\prime} \cap R_{v}\right)^{\infty}$. Since $i_{p_{0}^{\prime}}, s_{p_{0}^{\prime}} \in S^{\prime} \cap R_{v}$ by $\mathbf{I} 4$ the claim is proved.

Now ld $\Delta\left(p_{0}, p_{0}^{\prime}\right) \leq v$ thus, according to the specifications of Ritt's algorithms of reduction, there exists a power product $h$ of elements of $S^{\prime} \cap R_{v}$ such that $h \Delta\left(p_{0}, p_{0}^{\prime}\right) \equiv q \bmod \left(F_{v}^{\prime}\right)$. Since $\operatorname{rank} q \leq \operatorname{rank} \Delta\left(p_{0}, p_{0}^{\prime}\right)<v^{d}$ we have $q \in F_{v}^{\prime}$. Using the claim above, the lemma is proved.

\footnotetext{
${ }^{8}$ Actually, the lemma is false when $p, q$ are linear homogeneous differential polynomials, in one differential indeterminate $u$, with constant coefficients and when $\operatorname{ld} q=u$. In that case, the equation $p$ is lost. However, this does not matter for $q:=u=0$ makes superfluous all other linear homogeneous differential polynomials in $u$ alone and with constant coefficients.
} 
Proposition 39. - $\mathscr{I}(G):\left\{i_{q}, s_{q}\right\}^{\infty}=\mathscr{I}\left(G^{\prime}\right)$.

Proof. - The inclusion $\mathscr{I}(G):\left\{i_{q}, s_{q}\right\}^{\infty} \subset \mathscr{I}\left(G^{\prime}\right)$ is a corollary of Lemma 38. Let's prove the converse inclusion and first that $q \in \mathscr{I}(G)$. For this, we consider three cases:

First case: $q=q_{0}$ full-rem $A$ with $q_{0} \in P$. It is clear for $q_{0} \in \mathscr{I}(G)$, $A \subset \mathscr{I}(G)$ and $H_{A} \subset S$.

Second case: $q=\Delta\left(p_{0}, p_{0}^{\prime}\right)$ full-rem $A$ when $\left\{p_{0}, p_{0}^{\prime}\right\}$ is not a reduction critical pair. It comes from I5 and the fact that $A \subset \mathscr{I}(G)$ and $H_{A} \subset S$.

Third case: $q=\Delta\left(p_{0}, p_{0}^{\prime}\right)$ full-rem $A$ when $\left\{p_{0}, p_{0}^{\prime}\right\}$ is a reduction critical pair (with rank $p_{0}>\operatorname{rank} p_{0}^{\prime}$ ). It comes from the fact that $p_{0}^{\prime} \in \mathscr{I}(G)$ by $\mathbf{I} 2$ (applied to $G$ ), $p_{0} \in \mathscr{P}(D) \subset \mathscr{I}(G), A \subset \mathscr{I}(G)$ and $H_{A} \subset S$. Since $q \in \mathscr{I}(G)$, we have $A^{\prime} \subset \mathscr{I}(G)$. If $p \in \mathscr{P}\left(D^{\prime}\right)$ does not belong to $\mathscr{P}(D)$ then $p$ belongs to a reduction critical pair $\{p, q\} \in D^{\prime}$ with $p \in A$; thus $\mathscr{P}\left(D^{\prime}\right) \subset \mathscr{I}(G)$. The proof follows the fact that $P^{\prime} \subset P$ and $S^{\prime}=S \cup\left\{i_{q}, s_{q}\right\}$.

Proposition 40. - $G^{\prime}$ satisfies invariants I1, I4 and I6.

Proposition 41. - $G^{\prime}$ satisfies invariant I5.

Proof. - This comes from the fact that all the critical pairs in $D^{\prime}$ which are not reduction critical pairs have the form $\{p, q\}$ with $p, q \in A^{\prime} \subset \mathscr{I}\left(G^{\prime}\right)$, that $\mathscr{I}(G) \subset \mathscr{I}\left(G^{\prime}\right)$ and $G$ satisfies $\mathbf{I}$.

Proposition 42. - $G^{\prime}$ satisfies invariant I2.

Proof. - Invariant $\mathbf{I} 2$ is satisfied for all reduction critical pairs in $D^{\prime}$ which are not in $D$ since those critical pairs have the form $\{p, q\}$ with $\operatorname{rank} p>\operatorname{rank} q$ and $q \in A^{\prime}$. Invariant $\mathbf{I} 2$ is satisfied for all reduction critical pairs in $D^{\prime}$ which belong also to $D$ by Lemma 38 .

Lemma 43. - If $v$ is any derivative then every $\left\{p, p^{\prime}\right\} \in \operatorname{critical}-\operatorname{pairs}(A \cup$ $\{q\})$ which is such that $\operatorname{lcd}\left(\operatorname{ld} p, \operatorname{ld} p^{\prime}\right)<v$ is nearly solved by $G^{\prime}$.

Proof. - By induction on $v$. Basis of the induction: if $v$ is less than or equal to the minimum $v_{0}$ of $\operatorname{lcd}\left(\operatorname{ld} p, \operatorname{ld} p^{\prime}\right)$ for all $\left\{p, p^{\prime}\right\} \in \operatorname{critical}-\operatorname{pairs}(A \cup$ $\{q\})$ then the lemma is trivially satisfied. In the general case, let us assume that $v>v_{0}$ and (induction hypothesis) that every $\left\{p, p^{\prime}\right\} \in \operatorname{critical}-\operatorname{pairs}(A \cup$ $\{q\})$ such that $\operatorname{lcd}\left(\operatorname{ld} p, \operatorname{ld} p^{\prime}\right)<v$ is nearly solved by $G^{\prime}$.

First case: $p \neq q$ and $p^{\prime} \neq q$.

First subcase: if $\left\{p, p^{\prime}\right\}$ is solved by $G$ then, by Lemma 38, the critical pair $\left\{p, p^{\prime}\right\}$ is solved by $G^{\prime}$. It is thus nearly solved by $G^{\prime}$ according to A1.

Second subcase: if $\left\{p, p^{\prime}\right\} \in D^{*}$ then either it belongs to $D^{\prime}$ or it does not. In the former case, it is nearly solved by $G^{\prime}$ according to A2. In the latter, the triple $\left\langle p, q, p^{\prime}\right\rangle$ satisfies the hypotheses $\mathbf{H} \mathbf{1}$ to $\mathbf{H 3}$ of Proposition 27 and 
$\operatorname{lcd}\left(\operatorname{ld} p, \operatorname{ld} p^{\prime}\right)$ is a proper derivative of both $\operatorname{lcd}(\operatorname{ld} p, \operatorname{ld} q)$ and $\operatorname{lcd}\left(\operatorname{ld} p^{\prime}, \operatorname{ld} q\right)$. By the induction hypothesis and $\mathbf{A 3}$, the critical pair $\left\{p, p^{\prime}\right\}$ is nearly solved by $G^{\prime}$.

Third subcase: if $\left\{p, p^{\prime}\right\}=\left\{p_{0}, p_{0}^{\prime}\right\} \in D$ and $q=\Delta\left(p_{0}, p_{0}^{\prime}\right)$ full-rem $A$ then $\left\{p, p^{\prime}\right\}$ is solved by the differential system $A \cup\{q\}=0, S^{\prime} \neq 0$ (specifications of Ritt's reduction algorithms). By Lemma 36 and the fact that $q \in A^{\prime}$, the critical pair $\left\{p, p^{\prime}\right\}$ is solved by $G^{\prime}$. It is thus nearly solved by $G^{\prime}$ according to A1.

Second case: the critical pair is formed by $q$ and some $p \in A$.

First subcase: If $\{p, q\} \in D_{1} \subset D^{\prime}$ then $\{p, q\}$ is nearly solved by $G^{\prime}$ according to A2.

Second subcase: If $\{p, q\} \notin D^{\prime}$ then either it is solved by $G^{\prime}$ according to Proposition 26 or there exists a critical pair $\left\{p^{\prime}, q\right\} \in D_{1} \subset D^{\prime}$ such that the triple $\left\langle q, p^{\prime}, p\right\rangle$ satisfies the hypotheses $\mathbf{H 1}$ to $\mathbf{H 3}$ of Proposition 27. In this latter case, $\operatorname{lcd}(\operatorname{ld} p, \operatorname{ld} q)$ is a derivative of $\operatorname{lcd}\left(\operatorname{ld} p, \operatorname{ld} p^{\prime}\right)$ and, according to the first case considered above, the critical pair $\left\{p, p^{\prime}\right\} \in \operatorname{critical-pairs}(A)$ is solved by $G^{\prime}$. By $\mathbf{A} 3$ the critical pair $\{p, q\}$ is nearly solved by $G^{\prime}$.

Proposition 44. - $G^{\prime}$ satisfies I3.

Proof. - This follows Lemma 43 and the fact that $A^{\prime} \subset A \cup\{q\}$.

\section{$5.3 \quad$ Splittings}

When the completion process enlarges $A$ with a new equation $q=0$, the set $S$ is also enlarged with two inequations $i_{q} \neq 0, s_{q} \neq 0$. In order not to loose solutions of the current quadruple, we must also consider its solutions which cancel the initial or the separant of $q$. This we do by splitting cases as in Seidenberg's elimination algorithms. The argument relies on the differential analogue of Hilbert's theorem of zeros (Theorem 14).

Lemma 45. - If $A=0, S \neq 0$ is a differential system and $h$ is a differential polynomial then every solution of $A=0, S \neq 0$ is a solution of $A \cup\{h\}=$ $0, S \neq 0$ or a solution of $A=0, S \cup\{h\} \neq 0$ and conversely.

Corollary 46. $-\sqrt{[A]: S^{\infty}}=\sqrt{[A, h]: S^{\infty}} \cap \sqrt{[A]:(S \cup\{h\})^{\infty}}$.

Proof. - The corollary comes from Lemma 45 and the corollary 15 of the theorem of zeros.

Let's come back to the quadruples $G, G^{\prime}$ and to the differential polynomial $q$ of section 5.2. Denote $\operatorname{rank} q=v^{d}$. Let $q_{i}=q-i_{q} v^{d}$ and $q_{s}=d q-v s_{q}$. Denote

$$
\begin{aligned}
& G_{i}=\left\langle A, D^{*}, P^{*} \cup\left\{i_{q}, q_{i}\right\}, S\right\rangle, \\
& G_{s}=\left\langle A, D^{*}, P^{*} \cup\left\{s_{q}, q_{s}\right\}, S \cup\left\{i_{q}\right\}\right\rangle .
\end{aligned}
$$


Proposition 47. - $\mathscr{I}(G)=\mathscr{I}\left(G_{i}\right) \cap \mathscr{I}\left(G_{s}\right) \cap \mathscr{I}\left(G^{\prime}\right)$.

Proof. - Using Lemma 45, every solution of $\mathscr{F}(G)=0, S \neq 0$ is a solution of $\mathscr{F}(G) \cup\left\{i_{q}\right\}=0, S \neq 0$ (denoted by $\Sigma_{i}$ ) or a solution of $\mathscr{F}(G)=0, S \cup\left\{i_{q}\right\} \neq 0$ and conversely. Using Lemma 45 again, every solution of the latter system is a solution of $\mathscr{F}(G) \cup\left\{s_{q}\right\}=0, S \cup\left\{i_{q}\right\} \neq 0$ (denoted by $\Sigma_{s}$ ) or a solution of $\mathscr{F}(G)=0, S \cup\left\{i_{q}, s_{q}\right\} \neq 0$ (denoted by $\left.\Sigma^{\prime}\right)$ and conversely. The system $\Sigma_{i}$ (respectively $\Sigma_{s}$ ) has the same solutions as the quadruple $G_{i}$ (respectively $G_{s}$ ). By Proposition 39, the system $\Sigma^{\prime}$ has the same solutions as the quadruple $G^{\prime}$. The proposition follows now from Corollary 46.

Observe that if a differential polynomial $h$ does not divide zero modulo $\sqrt{[\Sigma]}$ then there is no need of splitting on $h$ since in that case $\sqrt{[\Sigma]}=$ $\sqrt{[\Sigma]: h^{\infty}}$. This is the case for instance if $h \in K(h \neq 0)$.

\subsubsection{The system $G_{i}$ satisfies all the invariants}

Note: the proofs are simpler variants of the ones given for $G^{\prime}$ in section 5.2. They rely on the fact that $q \in\left(i_{q}, q_{i}\right)$ and $\operatorname{rank} i_{q}, q_{i}<\operatorname{rank} q$. Therefore, since $q \in\left(s_{q}, q_{s}\right)$ and $\operatorname{rank} s_{q}, q_{s}<\operatorname{rank} q$ the same proofs hold for $G_{s}$; if $q=0$ then they hold for the quadruple $G^{*}=\left\langle A, D^{*}, P^{*}, S\right\rangle$ too.

Lemma 48. - If $v^{d}$ is any rank, $F=\left\{p \in \mathscr{F}(G) \mid \operatorname{rank} p \leq v^{d}\right\}$ and $F_{i}=\left\{p \in \mathscr{F}\left(G_{i}\right) \mid \operatorname{rank} p \leq v^{d}\right\}$ then $\left(F_{v}\right):\left(S \cap R_{v}\right)^{\infty} \subset\left(F_{i, v}\right):\left(S_{i} \cap R_{v}\right)^{\infty}$.

Proof. - We only have to consider two cases.

First case: $P^{*} \neq P$. More precisely, we assume $q=q_{0}$ full-rem $A$ with $q_{0} \in P$ and we prove that, if $\operatorname{rank} q_{0} \leq v^{d}$ then $q_{0} \in\left(F_{i, v}\right):\left(S_{i} \cap R_{v}\right)^{\infty}$. This comes from the fact that the elements of $A$ involved in the reduction process of $q_{0}$ have rank lower than or equal to that of $q_{0}$, the fact that $H_{A} \subset S \subset S^{\prime}$ and the fact that $q \in\left(i_{q}, q_{i}\right) \subset\left(F_{i, v}\right)$.

Second case: $\mathscr{P}\left(D^{*}\right) \neq \mathscr{P}(D)$. More precisely, we assume $q=\Delta\left(p_{0}, p_{0}^{\prime}\right)$ full-rem $A$ and $\left\{p_{0}, p_{0}^{\prime}\right\}$ is a reduction critical pair with $\operatorname{rank} p_{0}>\operatorname{rank} p_{0}^{\prime}$. We prove that, if rank $p_{0} \leq v^{d}$ then $p_{0} \in\left(F_{i, v}\right):\left(S_{i} \cap R_{v}\right)^{\infty}$. Claim: there exists a power product $h$ of elements of $S_{i} \cap R_{v}$ such that $h p_{0} \equiv \Delta\left(p_{0}, p_{0}^{\prime}\right) \bmod \left(F_{i, v}\right)$. Since $\left\{p_{0}, p_{0}^{\prime}\right\}$ is a reduction critical pair, there exists some derivation operator $\phi$ such that $\Delta\left(p_{0}, p_{0}^{\prime}\right)=p_{0}$ full-rem $\phi p_{0}^{\prime}$. Thus there exist $\alpha, \beta \in \mathbb{N}$ such that $i_{p_{0}^{\prime}}^{\alpha} s_{p_{0}^{\prime}}^{\beta} p_{0} \equiv \Delta\left(p_{0}, p_{0}^{\prime}\right) \bmod \left(\phi p_{0}^{\prime}\right)$. Using the fact that $G$ satisfies $\mathbf{I} 2$ and $\operatorname{rank} p_{0}^{\prime}<\operatorname{rank} p_{0} \leq v^{d}$ we see $p_{0}^{\prime} \in$ $\left(F_{i, v}\right):\left(S_{i} \cap R_{v}\right)^{\infty}$. Since ld $\phi p_{0}^{\prime}=\operatorname{ld} p_{0} \leq v$, we have $\phi p_{0}^{\prime} \in\left(F_{i, v}\right):\left(S_{i} \cap R_{v}\right)^{\infty}$. Since $i_{p_{0}^{\prime}}, s_{p_{0}^{\prime}} \in S_{i} \cap R_{v}$ by $\mathbf{I} 4$ the claim is proved.

Now ld $\Delta\left(p_{0}, p_{0}^{\prime}\right) \leq v$ thus, according to the specifications of Ritt's algorithms of reduction, there exists a power product $h$ of elements of $S_{i} \cap R_{v}$ 
such that $h \Delta\left(p_{0}, p_{0}^{\prime}\right) \equiv q \bmod \left(F_{i, v}\right)$. Since $\operatorname{rank} i_{q}, q_{i}<\operatorname{rank} \Delta\left(p_{0}, p_{0}^{\prime}\right)<v^{d}$ and $q \in\left(i_{q}, q_{i}\right)$ we have $q \in\left(F_{i, v}\right)$. Using the claim, the lemma is proved.

Proposition 49. - $G_{i}$ satisfies invariants I1, I4 and I6.

Proposition 50. - $G_{i}$ satisfies invariant I5.

Proof. - Because $\mathscr{I}(G) \subset \mathscr{I}\left(G_{i}\right)$ and $G$ satisfies invariant I5.

Proposition 51. - $G_{i}$ satisfies invariant I2.

PROOF. - This proposition is a corollary of Lemma 48.

Proposition 52. - $G_{i}$ satisfies invariant I3.

Proof. - Because of Lemma 48, all the critical pairs in $D$ solved by $G$ which still belong to $D^{*}$ are also solved by $G_{i}$. It suffices thus to show that if $q=\Delta\left(p_{0}, p_{0}^{\prime}\right)$ full-rem $A$ then $\left\{p_{0}, p_{0}^{\prime}\right\}$ is solved by $G_{i}$. This critical pair is solved by the differential system $A \cup\{q\}=0, S_{i} \neq 0$. Since $q \in\left(i_{q}, q_{i}\right) \subset \mathscr{F}\left(G_{i}\right)$ and $\operatorname{rank} i_{q}, q_{i}<\operatorname{rank} q$ the critical pair $\left\{p_{0}, p_{0}^{\prime}\right\}$ is solved by $G_{i}$.

\subsection{Proof of Theorem 28}

The axioms below define a partial ordering among quadruples. Let $G=$ $\left\langle A^{\prime}, D^{\prime}, P^{\prime}, S^{\prime}\right\rangle$ and $G=\langle A, D, P, S\rangle$ be two quadruples such that $\operatorname{rank} A$ and $\operatorname{rank} A^{\prime}$ are autoreduced.

O1 If $A^{\prime}<A$ then $G^{\prime}$ is said to be of lower rank than $G$.

O2 If $A^{\prime}=A$ and $D^{\prime}$ has fewer elements than $D$ then $G^{\prime}$ is said to be of lower rank than $G$.

O3 Assume $A^{\prime}=A$ and $D^{\prime}=D$. If there exists a differential polynomial $p \in$ $P$ and a finite set $E$ (possibly empty) of differential polynomials all of lower rank than $p$ such that $P^{\prime}=P \backslash\{p\} \cup E$ then $G^{\prime}$ is said to be of lower rank than $G$.

Lemma 53. - The ordering defined above is artinian (i.e. every strictly decreasing sequence of quadruples is finite).

Proof. - We assume there exists an infinite strictly decreasing sequence $\left(G_{n}\right)$ of quadruples and seek a contradiction. Denote $G_{n}=\left\langle A_{n}, D_{n}\right.$, $\left.P_{n}, S_{n}\right\rangle$. Since the ordering on autoreduced sets of differential polynomials is artinian, $\left(G_{n}\right)$ contains an infinite subsequence $\left(G_{i_{n}}\right)$ of quadruples such that all $A_{i}$ 's have the same rank. By a similar argument, $\left(G_{i_{n}}\right)$ contains itself 
an infinite subsequence $\left(G_{j_{n}}\right)$ of quadruples such that all $A_{j}$ 's have the same rank and all $D_{j}$ have the same number of elements. By an argument of graph theory [König, 1950, Satz 6.6] (i.e. every infinite locally finite ${ }^{9}$ tree contains a branch of infinite length) there exists (taken from the $P_{j}$ ) an infinite strictly decreasing sequence of differential polynomials. This cannot be for rankings are well-orderings. This final contradiction proves the Lemma.

Proof of Theorem 28. Differential systems are represented using quadruples. Let $G=\langle A, D, P, S\rangle$ be a quadruple of $R$ satisfying the invariant properties I1 up to I6. The initial system, coded $\left\langle\varnothing, \varnothing, P_{0}, S_{0}\right\rangle$ satisfies them. We assume inductively that the theorem holds for any quadruple $G^{\prime}<G$ satisfying the invariants. The induction is transfinite (Lemma 53).

Basis of the induction. Assume $D$ and $P$ are empty Applying the method described in section 5.1, one decides whether the differential system $A=$ $0, S \neq 0$ is consistent or not. It is discarded if it is inconsistent else one gets a regular differential system $\bar{A}=0, \bar{S} \neq 0$ such that $\mathscr{I}(G)=[\bar{A}]: \bar{S}^{\infty}$.

General case. Assume $D$ or $P$ not empty. Pick either a differential polynomial $q_{0} \in P$ or a critical pair $\left\{p_{0}, p_{0}^{\prime}\right\} \in D$. In the former case, let $P^{*}=P \backslash\left\{q_{0}\right\}$ let $D^{*}=D$ and $q=q_{0}$ full-rem $A$. In the latter let $P^{*}=P$, let $D^{*}=D \backslash\left\{\left\{p_{0}, p_{0}^{\prime}\right\}\right\}$ and $q=\Delta\left(p_{0}, p_{0}^{\prime}\right)$ full-rem $A$.

Assume that $q=0$ and let $G^{*}=\left\langle A, D^{*}, P^{*}, S\right\rangle$. The quadruple $G^{*}$ satisfies the invariants (cf. the note in section 5.3.1). Since $\mathscr{I}(G)=\mathscr{I}\left(G^{*}\right)$ and $G^{*}<G$ by $\mathbf{O} 2$ or $\mathbf{O} 3$ the quadruple $G^{*}$ can be disposed of by induction.

Assume $q \neq 0$. Let $G^{\prime}$ be any quadruple obtained following section 5.2. The quadruple $G^{\prime}$ satisfies the invariants and is of lower rank than $G$ according to O1. It can be disposed of by induction.

Denote $\operatorname{rank} q=v^{d}$. Let $q_{i}=q-i_{q} v^{d}$ and $q_{s}=d q-v s_{q}$. Form two quadruples $G_{i}$ and $G_{s}$ as in section 5.3. Since $q_{i}, q_{s}, i_{q}, s_{q}<q$ the quadruples $G_{i}$ and $G_{s}$ are both of lower rank than $G$ according to O2 or O3. They satisfy the invariants. They can be disposed of by induction.

The proof of the theorem is now completed by Proposition 47 .

\subsection{About the implementation}

The pseudo-code given in Figure 3 provides a method for constructing $D^{\prime}$ from $D_{0}$ and $D^{*}$ in the complete function given in Figure 2. Note that the first loop keeps the critical pairs which could be discarded using the analogue of Buchberger's first criterion (Proposition 26). Let's pseudo-quote [Becker

\footnotetext{
${ }^{9} \mathrm{~A}$ tree is said to be locally finite if only finitely many branches start from each of its nodes.
} 
and Weispfenning, 1991, page 231]: "if two or more critical pairs have the same least common derivative of leaders, so that there is a choice as to which one(s) should be deleted, then it is advantageous to try and keep one which will be discarded later by the analogue of Buchberger's first criterion. That way, one eventually gets rid of all of them."

\subsubsection{Avoiding splittings}

As stated in section 5.3, if a differential polynomial $h$ does not divide zero modulo $\mathfrak{p}=\sqrt{\left[P_{0}\right]: S_{0}^{\infty}}$ then there is nod need of splitting on $h$. Here is a way to apply this idea: before computing a decomposition of $\mathfrak{p}$ w.r.t. to some desired ranking $\mathscr{R}$ first compute a decomposition of $\mathfrak{p}$ w.r.t. another ranking $\mathscr{R}^{\prime}$ chosen heuristically so that the representation involves only few components. Afterwards, use it while computing the decomposition of $\mathfrak{p}$ w.r.t. $\mathscr{R}$ : each time the algorithm is about to split computations between (say) $h=0$ and $h \neq 0$, test whether $h$ is a divisor of zero modulo $\mathfrak{p}$. If $h$ is proved not to be a divisor of zero, the splitting can be avoided and the branch $h=0$ discarded. The differential polynomial $h \in \mathfrak{p}$ if and only if the branch $h \neq 0$ only leads to inconsistent regular differential systems. Such branches can therefore always be detected and discarded. If $h$ is proved to be a divisor of zero or if nothing can be proved then the splitting must be generated. The method above is particularly interesting when $\mathfrak{p}$ can be represented by a unique regular differential system $C=0$ which is orthonomic (i.e. all the initials and separants of $C$ belong to the base field of $R$ ). In that case (which turns out to happen quite often) $\mathfrak{p}=[C]$ is prime. No differential polynomial can divide zero modulo a prime ideal. The implementation of the RosenfeldGröbner algorithm in the diffalg package applies this improvement.

\subsubsection{Reducing the inequations}

It is interesting to keep $S$ partially reduced w.r.t. $A$ for inequations are usually small differential polynomials (for problems which can be handled): reducing them is not very CPU expensive and can point out inconsistencies. Note invariant $\mathbf{I} 4$ must then be changed and proofs modified.

\subsubsection{Linear equations}

If our implementation is given linear differential polynomials then the analogue of Buchberger's second criterion always applies ; moreover, no splittings are generated. In particular, if the given system is a set of non differential polynomials, coded as differential polynomials linear, homogeneous, in one differential indeterminate with constant coefficients then this implementation 
behaves exactly (up to the implementation overhead) as a good implementation of the Buchberger's algorithm (the one of Gebauer and Möller [1988]).

\section{Computing canonical representatives}

According to the results of the previous sections any regular differential ideal may be presented by a regular differential system and by its associated Gröbner basis. This was the choice in Boulier et al. [1995]. This representation is not only heavy but also non canonical for different regular differential systems may define the same regular differential ideal. In this section, we define better representatives of regular differential ideals that we call characteristic presentations. Theorem 59 then shows how to compute characteristic presentations from regular differential systems. The Rosenfeld-Gröbner algorithm (Theorem 60) can then be stated.

\subsection{Characteristic presentations}

The implication from left to right in the proof of the following theorem was already proved in [Boulier et al., 1995, Lemma 5, page 162].

THEOREM 54. - (canonicity theorem)

If $A_{1}=0, S_{1} \neq 0$ and $A_{2}=0, S_{2} \neq 0$ are two regular differential systems of some differential polynomial ring $R$ then $\left[A_{1}\right]: S_{1}^{\infty}=\left[A_{2}\right]: S_{2}^{\infty}$ iff $\left(A_{1}\right): S_{1}^{\infty}=\left(A_{2}\right): S_{2}^{\infty}$.

Proof. - The implication from left to right. We assume (H1) that $\left[A_{1}\right]: S_{1}^{\infty}=\left[A_{2}\right]: S_{2}^{\infty}$ and $(\mathbf{H 2})$ that $\left(A_{1}\right): S_{1}^{\infty} \neq\left(A_{2}\right): S_{2}^{\infty}$. We seek a contradiction. Denote $B_{1}=p_{1}<\cdots<p_{n}$ and $B_{2}=q_{1}<\cdots<q_{m}$ the Gröbner bases associated to the algebraic regular ideals. Apply $\mathbf{H 2}$ and assume $B_{1}<B_{2}$. There exists an index $i \leq n$ such that $p_{i}$ is not reduced to zero by $B_{2}$ and $p_{j}=q_{j}(1 \leq j<i)$. By $\mathbf{H 1}$ we have $p_{i} \in\left[A_{2}\right]: S_{2}^{\infty}$. By Corollary 12 and the fact that $p_{j}=q_{j}(1 \leq j<i)$, the differential polynomial $p_{i}$ is partially reduced w.r.t. $q_{1}, \ldots, q_{i-1}$. It is also partially reduced w.r.t. $q_{i}, \ldots, q_{m}$ for $\operatorname{ld} p_{i} \leq \operatorname{ld} q_{i}, \ldots, \operatorname{ld} q_{m}$ and $B_{2}$ is a Gröbner basis w.r.t. an elimination ordering defined by a ranking. By Corollary 12, $p_{i}$ is partially reduced w.r.t. $A_{2}$. By Rosenfeld's Lemma $p_{i} \in\left(A_{2}\right): S_{2}^{\infty}$. Contradiction.

The implication from right to left now. We assume $(\mathbf{H 1})$ that $\left(A_{1}\right): S_{1}^{\infty}=$ $\left(A_{2}\right): S_{2}^{\infty}$ and (H2) that $p \in\left[A_{1}\right]: S_{1}^{\infty}$. We claim $p \in\left[A_{2}\right]: S_{2}^{\infty}$. Let $q=\left(p\right.$ partial-rem $\left.A_{2}\right)$. There exists thus a power product $h$ of elements of $S_{2}$ such that $h p \equiv q$ modulo [ $\left.A_{2}\right]$. According to $\mathbf{H 1}$ we have $A_{2} \subset\left[A_{1}\right]: S_{1}^{\infty}$ thus $h p \equiv q$ modulo this latter ideal. Because of $\mathbf{H} 2$ we have $q \in\left[A_{1}\right]: S_{1}^{\infty}$. 
By Corollary 12 and $\mathbf{H 1}$ again $q$ is partially reduced w.r.t. $A_{1}$. By Rosenfeld's Lemma, it belongs to $\left(A_{1}\right): S_{1}^{\infty}=\left(A_{2}\right): S_{2}^{\infty}$. Let's summarize: ( $p$ partial-rem $\left.A_{2}\right) \in\left(A_{2}\right): S_{2}^{\infty}$. By Point 2 of Corollary $24, p \in\left[A_{2}\right]: S_{2}^{\infty}$.

In the next definition, the only purpose of conditions $\mathbf{C 2}$ and $\mathbf{C 3}$ is to ensure the canonicity property of characteristic presentations.

DEFINITION 55. - (characteristic presentations)

Let $A=0, S \neq 0$ be a consistent regular differential system of a differential polynomial ring $R$ for a ranking $\mathscr{R}$ and $B$ be the Gröbner basis associated to the regular algebraic ideal $(A): S^{\infty}$, computed in dimension zero.

$A$ differentially triangular set $C=p_{1}<\cdots<p_{n}$ is called a characteristic presentation of the regular differential ideal $[A]: S^{\infty}$ if it satisfies the following conditions:

C1 for any $p \in R$ we have $p \in[A]: S^{\infty}$ if and only if ( $p$ full-rem $\left.C\right)=0$,

C2 the set $C$ is a minimal differentially triangular subset of $B$,

C3 if $C^{\prime}=p_{1}^{\prime}<\cdots<p_{n}^{\prime}$ is another set which satisfies $\mathbf{C 1}$ and C2 and $i \leq n$ is the smallest index such that $p_{i} \neq p_{i}^{\prime}$ then the leading term ${ }^{10}$ of $p_{i}$ is less than the one of $p_{i}^{\prime}$.

A characteristic presentation $C$ of a regular differential ideal $[A]: S^{\infty}$ is not exactly a characteristic set in the sense of Ritt of the ideal since it is not autoreduced. However, it has the same rank as the characteristic sets of the ideal and it could easily be made autoreduced by performing a few reductions. We have $[A]: S^{\infty}=[C]: H_{C}^{\infty}$. Remark also that a characteristic set of $[A]: S^{\infty}$ is not necessarily a characteristic presentation of this ideal since it may reduce to zero more than the ideal.

THEOREM 56. - (canonicity of characteristic presentations)

If it exists, the characteristic presentation of a regular differential ideal is a canonical representative of this ideal (it only depends on the ideal and on the ranking).

Proof. - It is an easy consequence of Theorem 54, conditions C2 and C3 and the canonicity property of reduced Gröbner bases.

Here is an algorithm to extract a minimal differential triangular subset $C$ from the associated Gröbner basis $B$ of a consistent regular differential system $A=0, S \neq 0$ : for each derivative $v$ which is the leader of some element of $A$, pick from $B$ a differential polynomial with leader $v$ and with minimal degree in $v$ (among the elements of $B$ whose leader is $v$ ).

\footnotetext{
${ }^{10}$ The term ordering used is the elimination one given by the ranking.
} 
If $C$ is such a set of differential polynomials then $C$ is a triangular subset of $B$. By Lazard's Lemma, a derivative $v$ is the leader of some element of $B$ if and only if it is the leader of some element of $A$. Thus $C$ is a minimal triangular subset of $B$. Since $A$ is differentially triangular, so is $C$.

Lemma 57. - (algorithmic test for condition $\mathbf{C 1 )}$

Let $A=0, S \neq 0$ be a consistent regular differential system of a differential polynomial ring $R$ for a ranking $\mathscr{R}$ and $B$ be its associated Gröbner basis.

If $C$ is a minimal differentially triangular subset of $B$ and no element of $H_{C}$ is a divisor of zero modulo $(A): S^{\infty}$ then $C$ satisfies $\mathbf{C 1}$.

Proof. - By the hypothesis and Point 3 of Corollary 24, no element of $H_{C}$ is a divisor of zero modulo $[A]: S^{\infty}$. Since $C \subset[A]: S^{\infty}$, the set $C$ only reduces to zero elements of this differential ideal.

By the corollary of Lazard's Lemma, a derivative $v$ is the leader of some element of $C$ if and only if it is the leader of some element of $B$. Thus if $p$ is a non zero differential polynomial reduced in the sense of Ritt w.r.t. $C$ then $p$ is partially reduced w.r.t. $A$ on one hand; on the other hand, the terms of $p$ are not divisible by the leading terms of the elements of $B$ thus $p$ is irreducible by the Gröbner basis $B$ and $p \notin(B)=(A): S^{\infty}$. By Rosenfeld's Lemma, $p \notin[A]: S^{\infty}$. Thus every element of $[A]: S^{\infty}$ is reduced to zero by $C$.

Lemma 58. - Let $A=0, S \neq 0$ be a consistent regular differential system of a differential polynomial ring $R$ for a ranking $\mathscr{R}, B$ be its associated Gröbner basis and $C$ be a minimal differentially triangular subset of $B$.

An element $h \in H_{C}$ is a divisor of zero modulo $(B)$ if and only if the reduced Gröbner basis of $(B): h^{\infty}$ (computed in dimension zero) is different from $B$.

Proof. - First note $h \notin(B)$ for $h$ is irreducible by $B$. Now, the ideal $(B)$ is radical by Lazard's Lemma; the prime ideals which are minimal over $(B): h^{\infty}$ are the minimal prime ideals of $(B)$ which do not contain $h$; a polynomial $h$ is a divisor of zero modulo a radical ideal $\mathfrak{r}$ if and only if it belongs to some but not all of the prime ideals which are minimal over $\mathfrak{r}$; reduced Gröbner bases are canonical representatives of the ideals they generate.

THEOREM 59. - (computing characteristic presentations)

If $A=0, S \neq 0$ is a consistent regular differential system for a ranking $\mathscr{R}$ of a differential polynomial ring $R$ then it is possible to compute finitely many regular differential ideals given by characteristic presentations $C_{i}(i=$ $1, \ldots, n)$ such that

$$
[A]: S^{\infty}=\left[C_{1}\right]: H_{C_{1}}^{\infty} \cap \cdots \cap\left[C_{n}\right]: H_{C_{n}}^{\infty} .
$$


This decomposition does not contain redundant components w.r.t. $[A]: S^{\infty}$. Operations needed are addition, multiplication and equality test with zero in the base field of $R$.

Proof. - Denote $\mathfrak{r}=[A]: S^{\infty}$ and $B$ the Gröbner basis associated to $(A): S^{\infty}$. The paragraph above Lemma 57 shows how to extract minimal differentially triangular subsets $C$ from $B$. Lemmas 57 and 58 show how to test if one of them is a characteristic presentation of $\mathfrak{r}$.

The proof is an induction on the number of prime components of $(B)$. If $(B)$ is prime and $C$ is a minimal differentially triangular subset of $B$ then no element of $H_{C}$ divides zero modulo $(B)$ hence $C$ satisfies $\mathbf{C 1}$.

Assume $(A): S^{\infty}$ admits no characteristic presentation. Let $C$ be a minimal differentially triangular subset of $B$ and $p \in H_{C}$ be a divisor of zero modulo $(B)$. We have $\mathfrak{r}=\mathfrak{r}_{1} \cap \mathfrak{r}_{2}$ where $\mathfrak{r}_{1}=\sqrt{[A \cup\{p\}]: S^{\infty}}$ and $\mathfrak{r}_{2}=\mathfrak{r}: p^{\infty}$.

Both $\mathfrak{r}_{1}$ and $\mathfrak{r}_{2}$ have fewer components than $\mathfrak{r}$. The latter ideal is a regular differential system whence is disposed of by induction. Using Theorem 28, one can compute a representation of $\mathfrak{r}_{1}$ as an intersection of regular differential ideals. Moreover, one can manage to compute an irredundant intersection by using the technique described in paragraph 5.5.1

The method described in the proof above is the one applied in the diffalg package. It is not very efficient. The Lextriangular algorithm of Lazard [1992] (see also [Moreno Maza, 1997]) would be much more efficient and would even permit us to completely avoid the use of Gröbner bases.

\subsubsection{An example}

Some regular differential ideals (quite unusual in practice) have no characteristic presentation. An example is given by the following triangular set $A$, for the elimination ordering $x_{5}>\cdots>x_{1}$. The example is purely algebraic but can be easily transformed into a differential one.

$$
A\left\{\begin{array}{l}
p_{3}=\left(\left(x_{2}^{2}+x_{1}\right) x_{5}+x_{4}^{2}+x_{3}\right)\left(x_{2} x_{5}+x_{4}\right), \\
p_{2}=x_{4}\left(x_{4}^{2}+x_{3}\right) \\
p_{1}=x_{2}\left(x_{2}^{2}+x_{1}\right)
\end{array}\right.
$$

Below is the reduced Gröbner basis $B$ (computed over $\mathbb{Q}$ ) of the ideal $(A): S_{A}^{\infty}$ for the elimination ordering $x_{5}>\cdots>x_{1}$.

$$
B\left\{\begin{array}{l}
b_{5}=x_{5}, \\
b_{4}=x_{4}\left(x_{4}^{2}+x_{3}\right), \\
b_{3}=x_{1} x_{4}^{2}+\left(x_{2}^{2}+x_{1}\right) x_{3}, \\
b_{2}=x_{2} x_{4} \\
b_{1}=x_{2}\left(x_{2}^{2}+x_{1}\right) .
\end{array}\right.
$$


It contains only one minimal triangular subset $\left\{b_{1}, b_{2}, b_{5}\right\}$, which is not a characteristic presentation of $\mathfrak{r}=(A): S_{A}^{\infty}$ since the initial $x_{2}$ of $b_{2}$ is a divisor of zero modulo $\mathfrak{r}$. Moreover, $\mathfrak{r}$ contains no differentially triangular subset satisfying $\mathbf{C 1}$. Let us assume the existence of such a set $C$ and seek a contradiction. This set reduces $b_{2}$ to zero. So it contains either a polynomial $p \in \mathfrak{r} \cap K\left[x_{1}, x_{2}\right]$ of degree 1 in $x_{2}$ (impossible) or a polynomial $p \in \mathfrak{r} \cap$ $K\left[x_{1}, \ldots, x_{4}\right]$ of degree 1 in $x_{4}$, say $p=a_{1} x_{4}+a_{0}$. In this latter case, $p \in\left(b_{1}, b_{2}\right)$ is a multiple of $x_{2}$ and so is $a_{1}$ which is thus a divisor of zero modulo $\mathfrak{r}$ (contradiction). According to Theorem 59, the regular ideal can be decomposed as an intersection of regular ideals which admit characteristic presentations. The ideal $\mathfrak{r}=(A): S_{A}^{\infty}$ is decomposed as the intersection $\mathfrak{r}=\mathfrak{r}_{1} \cap \mathfrak{r}_{2}$ where $\mathfrak{r}_{1}=\mathfrak{r}: x_{2}^{\infty}$ and $\mathfrak{r}_{2}=\mathfrak{r}+\left(x_{2}\right)$. Here are characteristic presentations of these two ideals.

$$
\mathfrak{r}_{1}\left\{\begin{array} { l } 
{ x _ { 5 } , } \\
{ x _ { 4 } , } \\
{ x _ { 2 } ^ { 2 } + x _ { 1 } ; }
\end{array} \quad \mathfrak { r } _ { 2 } \left\{\begin{array}{l}
x_{5}, \\
x_{4}^{2}+x_{3}, \\
x_{2} .
\end{array}\right.\right.
$$

\subsection{The main theorem}

THEOREM 60. - (the Rosenfeld-Gröbner algorithm)

If $P_{0}=0, S_{0} \neq 0$ is a differential system of a differential polynomial ring $R$ then it is possible to compute finitely many regular differential systems given by characteristic presentations $C_{i}(i=1, \ldots, n)$ such that

$$
\mathfrak{p}=\sqrt{\left[P_{0}\right]: S_{0}^{\infty}}=\left[C_{1}\right]: H_{C_{1}}^{\infty} \cap \cdots \cap\left[C_{n}\right]: H_{C_{n}}^{\infty} .
$$

Operations needed are addition, multiplication, differentiation and equality test with zero in the base field of $R$. This decomposition may contain components redundant w.r.t. $\mathfrak{p}$. It provides a normal simplifier for the equivalence relation modulo this ideal i.e.

$$
p \in \mathfrak{p} \Longleftrightarrow p \text { full-rem } C_{i}=0 \quad(1 \leq i \leq n) .
$$

Proof. - The first claim is proved by Theorems 28 and 59. The property of being a normal simplifier is a consequence of condition C1 of definition 55.

By applying a primary decomposition algorithm over the regular decomposition of $\mathfrak{p}$, one would get a (redundant) differential prime decomposition of $\mathfrak{p}$ (see a remark below Theorem 25). This algorithm would probably be much more efficient than the characteristic sets algorithm of Ritt [1950] and 
would provide the same result. Remark that decomposition of radical differential ideals in regular differential ideals does not depend on the base field whereas the decomposition in prime differential ideals does. The computed representation of a radical differential ideal $\mathfrak{p}$ is not canonical because of the regular components which may be redundant w.r.t. $\mathfrak{p}$. Moreover, even if $\mathfrak{r}$ is a regular differential ideal which is not redundant w.r.t. $\mathfrak{p}$, there may exist among the minimal differential prime components of $\mathfrak{r}$ some differential ideals redundant w.r.t. p. Deciding whether a regular differential ideal is redundant or not w.r.t. a decomposition of type (14) is related to a famous open problem in differential algebra [Kolchin, 1973, page 166]. The computed representation of $\mathfrak{p}$ is therefore not a canonical simplifier for the equivalence relation modulo $\mathfrak{p}$. However, being a normal simplifier is enough for deciding whether two given differential polynomials $p$ and $q$ are equivalent modulo $\mathfrak{p}$ for $p \equiv q$ if and only if $p-q \equiv 0$ modulo $\mathfrak{p}$. In the case of differential ideals generated by only one differential polynomial, the problem of the computation of the minimal prime decomposition is solved by the Low Power Theorem [Kolchin, 1973, chapter IV, section 15], much studied by Ritt [1950] and Levi [1945]. See also [Hubert, 1997] for an implementation of this theorem based on the Rosenfeld-Gröbner algorithm and a generalization of it to regular differential decompositions.

\section{$7 \quad$ Formal power series solutions of regular dif- ferential ideals}

The content of this section is a variant of Seidenberg's results [Seidenberg, 1956, Theorem 11, page 59] [Seidenberg, 1958, Embedding Theorem] and 1969. We give proofs for the sake of completeness and because the hypotheses of Seidenberg's theorems are slightly different from ours. This section was also partly inspired by Péladan-Germa [1997].

Let $A=0, S \neq 0$ be a differential system of a differential polynomial ring $R=K\left\{u_{1}, \ldots, u_{n}\right\}$ and $R_{0}$ be the ring of the differential polynomials partially reduced w.r.t. $A$. Let $\phi_{0}$ be any algebraic solution of $A=0, S \neq 0$, viewed as a non differential system of $R_{0}$. The solution $\phi_{0}$ defines a $K$-algebra homomorphism $\phi_{0}: R_{0} \rightarrow G$ where $G$ is some field extension of $K$. Note that $\phi_{0}$ maps the elements of $S$ to nonzero elements of $G$. We prove first (Proposition 62) that $\phi_{0}$ extends to a unique solution $\phi$ of the differential ideal $[A]: S^{\infty}$. Then we prove $\phi$ is uniquely defined (Proposition 63 ) and provides the coefficients of a formal power series solution of $[A]: S^{\infty}$ (Proposition 65).

Let $v \in \Theta U$ be a derivative and let $p=v$ partial-rem $A$. There exists 
then a power product $h$ of elements of $S$ and a differential polynomial $p \in R_{0}$ such that

$$
h v \equiv p \bmod [A]
$$

We define $\phi(v)=\phi_{0}(p) / \phi_{0}(h)$.

Lemma 61. - The map $\phi$ is well defined (i.e. the definition does not depend on the differential polynomials $h$ and $p$ ).

Proof. - Let $h, p$ be the differential polynomials defined in congruence (15). Assume there exists another power product $h^{\prime}$ of elements of $S$ and another differential polynomial $p^{\prime} \in R_{0}$ such that $h^{\prime} v \equiv p^{\prime} \bmod [A]$. We have $h p^{\prime}-h^{\prime} p \in[A]: S^{\infty} \cap R_{0}$. Since $A=0, S \neq 0$ is a regular differential system, Rosenfeld's Lemma applies and $h p^{\prime}-h^{\prime} p \in(A): S^{\infty}$ whence $\phi_{0}(p) / \phi_{0}(h)=\phi_{0}\left(p^{\prime}\right) / \phi_{0}\left(h^{\prime}\right)$.

The map $\phi$ extends to a unique $K$-algebra homomorphism $K[\Theta U] \rightarrow G$ that we denote by $\phi$ also.

Proposition 62. - If $p \in[A]: S^{\infty}$ then $\phi(p)=0$.

Proof. - First observe that if $p$ is a proper derivative of some element of $A$ then there exists a possible partial reduction such that $p$ partial-rem $A=0$; according to Lemma 61 we have $\phi(p)=0$. Now, if $p \in[A]: S^{\infty}$ then $\bar{p}=(p$ partial-rem $A) \in(A): S^{\infty}$ by Rosenfeld's Lemma whence $\phi(\bar{p})=0$. Moreover, there exists a power product $h$ of elements of $S$ such that $h p-\bar{p}$ is equal to a linear combination of proper derivatives of elements of $A$; thus $\phi(p)=\phi(\bar{p}) / \phi(h)=0$.

Proposition 63. - The homomorphism $\phi$ is the unique K-algebra homomorphism extending $\phi_{0}$ which maps $[A]: S^{\infty}$ to zero.

Proof. - Assume there exists another homomorphism $\phi^{\prime}$ extending $\phi_{0}$ which maps $[A]: S^{\infty}$ to zero. Let $v \in \Theta U$ be a derivative and $h, p$ be the differential polynomials defined in congruence (15). We have $\phi^{\prime}(v)=$ $\phi_{0}(p) / \phi_{0}(h)=\phi(v)$.

If $\alpha=\left(\alpha_{1}, \ldots, \alpha_{m}\right) \in \mathbb{N}^{m}$ is a multi-index, and $\eta=\left(\eta_{1}, \ldots, \eta_{m}\right) \in G^{m}$, then let $\alpha !=\prod_{i=1}^{m} \alpha_{i}$ ! and $(x-\eta)^{\alpha}=\left(x_{1}-\eta_{1}\right)^{\alpha_{1}} \cdots\left(x_{m}-\eta_{m}\right)^{\alpha_{m}}$ and $\delta^{\alpha}=\delta_{1}^{\alpha_{1}} \cdots \delta_{m}^{\alpha_{m}}$. To each differential indeterminate $u \in U$ we can associate a formal power series ( $\eta$ is the point of expansion of the series):

$$
\bar{u}=\sum_{\alpha \in \mathbb{N}^{m}} \frac{\phi\left(\delta^{\alpha} u\right)}{\alpha !}(x-\eta)^{\alpha} .
$$

The derivations defined over $R$ act over such a formal power series according to the rules:

$$
\delta_{i} x_{i}=1, \quad \delta_{i} x_{j}=0, \quad(i \neq j) .
$$


Lemma 64. - The substitution $u \rightarrow \bar{u}$ defines a differential homomorphism of $K$-algebra $R \rightarrow G[[x-\eta]]$.

We omit the proof which is purely computational.

Proposition 65 . - The n-tuple $\left(\bar{u}_{1}, \ldots, \bar{u}_{n}\right)$ is a differential solution of the differential ideal $[A]: S^{\infty}$.

Proof. - Using Lemma 64, for any differential polynomial $p \in R$ we have

$$
p\left(\bar{u}_{1}, \ldots, \bar{u}_{n}\right)=\sum_{\alpha \in \mathbb{N}^{m}} \frac{\phi\left(\delta^{\alpha} p\right)}{\alpha !}(x-\eta)^{\alpha}
$$

hence $p\left(\bar{u}_{1}, \ldots, \bar{u}_{n}\right)=0$ if and only if $\phi\left(\delta^{\alpha} p\right)=0$ for each $\alpha \in \mathbb{N}^{m}$. Since $\phi$ maps $[A]: S^{\infty}$ to zero and $[A]: S^{\infty}$ is a differential ideal, for every $p \in[A]: S^{\infty}$ and every $\alpha \in \mathbb{N}^{m}$ we have $\phi\left(\delta^{\alpha} p\right)=0$ whence $p\left(\bar{u}_{1}, \ldots, \bar{u}_{n}\right)=0$.

A regular differential ideal may have a formal power series solution for initial conditions which annihilate some elements of $S$. The simplest example is probably $u_{x}^{2}-4 u=0$ with $u_{x} \neq 0$ and $\phi_{0}\left(u_{x}\right)=0$ (the solution being $\left.u(x)=x^{2}\right)$. The formal power series defined here do not belong to $G[[x]]$ but to $G[[x-\eta]]$.

\section{Examples}

We detail the resolution of the system presented in the introduction with the help of the diffalg package of MAPLE V.

$$
\Sigma \begin{cases}u_{x}^{2}-4 u & =0, \\ u_{x y} v_{y}-u+1 & =0, \\ v_{x x}-u_{x} & =0 .\end{cases}
$$

The following instructions load the package and store in $R$ the differential polynomial ring $\mathbb{Q}(x, y)\{u, v\}$ endowed with derivations w.r.t. $x$ and $y$ and an orderly ranking over $\Theta\{u, v\}$ such that

1. if $\operatorname{ord}(\theta)=\operatorname{ord}(\varphi)$ then $\theta u>\varphi v$

2. if $\operatorname{ord}(\theta)=\operatorname{ord}(\varphi)$ and $\theta>\varphi$ for the lexical order $x>y$ then $\theta u>\varphi u$ (idem for $v$ ).

$>$ with ( diffalg) :

$>\mathrm{R}:=$ differential_ring ( derivations $=[\mathrm{x}, \mathrm{y}]$, ranking $=[\mathrm{u}, \mathrm{v}]]$ ):

The Rosenfeld-Gröbner algorithm is called and returns a list (understand "intersection") of regular differential ideals presented by characteristic sets. The ideals are stored in MAPLE tables. Only the names of the tables (i.e. "regular") get printed. Over this example, the list only involves one table. 


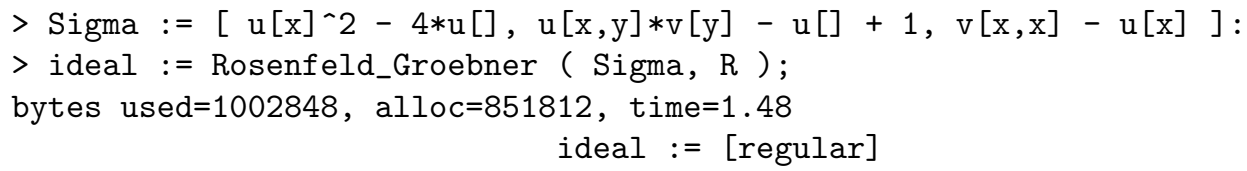

The following instruction displays the characteristic presentation of the regular ideal as rewrite rules for Ritt's reduction algorithms: let $p$ be a differential polynomial with rank $v^{d}$; then $p=a_{d} v^{d}+a_{d-1} v^{d-1}+\cdots+a_{0}$ for some differential polynomials $a$ 's (the initial of $p$ is $a_{d}$ ). the differential polynomial $p$ is displayed as

$$
v^{d}=-\frac{a_{d-1} v^{d-1}+\cdots+a_{0}}{a_{d}} .
$$

$>$ rewrite_rules ( ideal [1] );

$$
\left[v_{x, x}=2 \frac{u_{y} v_{y}}{-1+u}, u_{x}=2 \frac{u_{y} v_{y}}{-1+u}, u_{y}^{2}=2 u, v_{y}^{2}=\frac{1}{2} u^{2}-u+\frac{1}{2}\right]
$$

Looking at the leaders of the differential polynomials we see that there are only three derivatives (i.e. $u, v$ and $v_{x}$ ) which are not derivatives of the leader of any equation of the characteristic presentation. The solutions of $\Sigma$ depend therefore on three arbitrary constants (the symbols starting with underscores denote initial conditions).

> initial_conditions ( ideal [1] );

$$
\left[-C u,{ }_{-} C v,{ }_{-} C v_{-} x\right]
$$

The following function call computes two objects from the computed representation which give us formal power series solutions of $\Sigma$.

1. a "generic" formal power series solution of $\Sigma$ expanded at the origin and up to order 100 (the series turn out to be a polynomial); this is the returned value of the function call,

2. a triangular system of non differential polynomial equations and inequations over the initial conditions (this is returned in the output parameter syst).

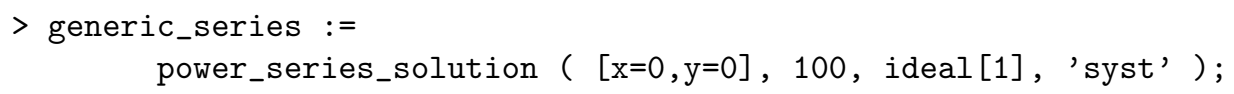




$$
\begin{aligned}
& \text { generic_series }:=\left[u(x, y)={ }_{-} C u+2 \frac{x_{-} C u_{-} y{ }_{-} C v_{-} y}{-1+{ }_{-} C u}+y_{-} C u_{-} y\right. \\
& -\frac{1}{2} \frac{x^{2}\left(-128_{-} C v_{-} y_{-} C u^{3}+384_{-} C v_{-} y_{-} C u^{2}-384_{-} C v_{-} y_{-} C u+128_{-} C v_{-} y\right)}{64_{-} C v_{-} y y_{-} C u^{3}-192_{-} C v_{-} y_{-} C u^{2}+192_{-} C v_{-} y_{-} C u-64_{-} C v_{-} y} \\
& -\frac{x y\left(48_{-} C u^{2}-48_{-} C u-16_{-} C u^{3}+16\right)}{16_{-} C v_{-} y-32_{-} C v_{-} y_{-} C u+16_{-} C v_{-} y_{-} C u^{2}}+\frac{1}{2} y^{2} \text {, } \\
& v(x, y)={ }_{-} C v+x_{-} C v_{-} x+y_{-} C v_{-} y+\frac{x^{2}{ }_{-} C u_{-} y{ }_{-} C v_{-} y}{-1+{ }_{-} C u}+x y_{-} C u_{-} y \\
& -\frac{1}{8} \frac{y^{2}\left(-2 z_{-} C u_{-} C u_{-} y+2_{-} C u_{-} y\right)}{{ }_{-} C v_{-} y} \\
& -\frac{1}{6} \frac{x^{3}\left(-128_{-} C v_{-} y_{-} C u^{3}+384_{-} C v_{-} y_{-} C u^{2}-384_{-} C v_{-} y_{-} C u+128_{-} C v_{-} y\right)}{64_{-} C v_{-} y_{-} C u^{3}-192_{-} C v_{-} y_{-} C u^{2}+192_{-} C v_{-} y_{-} C u-64_{-} C v_{-} y} \\
& -\frac{1}{2} \frac{x^{2} y\left(48_{-} C u^{2}-48_{-} C u-16_{-} C u^{3}+16\right)}{16_{-} C v_{-} y-32_{-} C v_{-} y_{-} C u+16_{-} C v_{-} y_{-} C u^{2}} \\
& -\frac{1}{2} \frac{x y^{2}\left(-128_{-} C v_{-} y_{-} C u^{2}+256_{-} C v_{-} y_{-} C u-128_{-} C v_{-} y\right)}{128_{-} C v_{-} y_{-} C u^{2}-256_{-} C v_{-} y_{-} C u+128_{-} C v_{-} y} \\
& \left.-\frac{1}{6} \frac{y^{3}\left(-128_{-} C u^{3}+384_{-} C u^{2}-384_{-} C u+128\right)}{256 \_C v_{-} y_{-} C u^{2}-512_{-} C v_{-} y_{-} C u+256 \_C v_{-} y}\right]
\end{aligned}
$$

> syst;

$$
\begin{aligned}
& {\left[-_{-} C v_{-} x x+{ }_{-} C v_{-} x x x_{-} C u-2 \__{-} C u_{-} y \_C v_{-} y=0\right. \text {, }} \\
& { }_{{ }_{-} C} C u_{-} x+{ }_{-} C u_{-} x x_{-} C u-2{ }_{-} C u_{-} y{ }_{-} C v_{-} y=0,-2{ }_{-} C u+{ }_{-} C u_{-} y^{2}=0 \text {, } \\
& \left.-_{-} C u^{2}+2{ }_{-} C u+2{ }_{-} C v_{-} y^{2}-1=0,{ }_{-} C v_{-} y \neq 0,{ }_{-} C u_{-} y \neq 0,-1+{ }_{-} C u \neq 0\right]
\end{aligned}
$$

According to section 7, every solution of syst furnishes a unique formal power series solution of $\Sigma$. According to Lazard's Lemma ${ }_{-} C u,{ }_{-} C v$ and ${ }_{-} C v_{-} x$ furnish a family of abitrary parameters. Let's take _ $C u=5,{ }_{-} C v=421$ and _ $C v_{-} x=\pi$. The specialized system has now only finitely many solutions. Here is one of them, computed from bottom up.

$$
\begin{aligned}
\text { algebraic_solution }:= & { }_{-} C v_{\_} x x=\sqrt{10} \sqrt{2},{ }_{-} C u_{-} x=\sqrt{10} \sqrt{2},{ }_{-} C u_{-} y=\sqrt{10}, \\
& { }_{-} C v_{-} y=2 \sqrt{2},{ }_{-} C u=5,{ }_{-} C v=421,{ }_{-} C v_{-} x=\pi
\end{aligned}
$$

The corresponding solutions of $\Sigma$ are obtained by specializing the formal power series of generic_series at algebraic_solution.

$>$ subs ( algebraic_solution, generic_series ); 


$$
\begin{gathered}
{\left[u(x, y)=5+x \sqrt{10} \sqrt{2}+y \sqrt{10}+x^{2}+x y \sqrt{2}+\frac{1}{2} y^{2}\right.} \\
v(x, y)=421+x \pi+2 y \sqrt{2}+\frac{1}{2} x^{2} \sqrt{10} \sqrt{2}+x y \sqrt{10}+\frac{1}{4} y^{2} \sqrt{10} \sqrt{2} \\
\left.+\frac{1}{3} x^{3}+\frac{1}{2} x^{2} y \sqrt{2}+\frac{1}{2} x y^{2}+\frac{1}{12} y^{3} \sqrt{2}\right]
\end{gathered}
$$

\subsection{Lie symmetries with automatic discussion}

This example consists in solving a system of linear partial differential equations depending on a parameter. By splitting cases, the Rosenfeld-Gröbner algorithm actually discusses the solutions w.r.t. the parameter. The example and a part of its analysis are borrowed from Reid [1991]. It deals with Lie symmetries of differential equations. See [Olver, 1993] and [1995] for the mathematical theory. The following differential equation is a variant of the wave equation. The symbol $H$ denotes an arbitrary function of $u(x, y)$ (i.e. a parameter of the differential equation).

$$
\mathrm{E}_{H}: \quad \frac{\partial^{2}}{\partial x^{2}} u(x, y)=\frac{\partial^{2}}{\partial y^{2}} u(x, y)+H(u(x, y)) \frac{\partial}{\partial y} u(x, y)
$$

We are concerned with the Lie symmetries of the equation $\left(\mathrm{E}_{H}\right)$. Indeed, the graph of a solution of the equation $\left(\mathrm{E}_{H}\right)$ is a set of points $(x, y, u) \in \mathbb{R}^{3}$; a Lie symmetry of this equation is a transformation (a local diffeomorphism) which maps the graphs of solutions to the graphs of other solutions:

$$
\left\{\begin{array}{l}
X=\varphi_{1}(x, y, u) \\
Y=\varphi_{2}(x, y, u) \\
U=\varphi_{3}(x, y, u)
\end{array}\right.
$$

We are looking for vector fields

$$
V=V^{1}(x, y, u) \frac{\partial}{\partial x}+V^{2}(x, y, u) \frac{\partial}{\partial y}+V^{3}(x, y, u) \frac{\partial}{\partial u}
$$

whose flows are the desired symmetries. The set of these vector fields form a Lie algebra i.e. a vector space endowed with a Lie bracket. With the help of the liesymm package of MAPLE, we build a system $\Sigma_{H}$ of linear partial derivatives equations in the three differential indeterminates $V^{1}, V^{2}$ and $V^{3}$ and derivations w.r.t. $x, y$ and $u$.

$$
\begin{aligned}
\Sigma_{H} & =\left[V_{x x}^{1}-H V_{y}^{1}-2 V_{x u}^{3}-V_{y y}^{1}, V_{x x}^{2}-V_{y y}^{2}+H V_{y}^{2}+V^{3} H_{u}+2 V_{y u}^{3},\right. \\
& V_{x x}^{3}-H V_{y}^{3}-V_{y y}^{3}, V_{u u}^{1}, V_{u u}^{2},-2 V_{x u}^{1}+V_{u u}^{3}, V_{u}^{2}, V_{u}^{1}, V_{x}^{1}-V_{y}^{2}, V_{y u}^{2}-V_{x u}^{1}, \\
& \left.V_{x}^{2}-V_{y}^{1}, V_{x u}^{2}-V_{y u}^{1}\right]
\end{aligned}
$$


Derivatives of the parameter $H$ appear in the coefficients of the linear differential equations. We enlarge the system with the two following equations, to express the fact that $H$ only depends on $u$.

$$
H_{x}=0, H_{y}=0 .
$$

We want to discuss w.r.t. $H$ the structure of the Lie algebra (in particular, its dimension as a vector space). For this reason, we consider $\Sigma_{H}$ as a system of polynomial differential equations in four differential indeterminates $V^{1}, V^{2}$, $V^{3}$ and $H$ and we call the Rosenfeld-Gröbner algorithm with a ranking which eliminates the $V$ 's. By splitting cases, the Rosenfeld-Gröbner algorithm discusses the structure of the Lie algebra w.r.t. $H$. Four regular systems are generated. In the paragraphs below, computations of regular differential systems and Taylor expansions of solutions are performed using the diffalg package. Outputs are pretty printed. Taylors expansions are computed in the neighborhoud of $x=0, y=0, u=0$. The symbols starting with a $C$ denote the constants appearing in these developments (e.g. $\mathrm{CH}=\mathrm{H}(0,0,0), \mathrm{CH}_{u}=$ $\left.H_{u}(0,0,0), \ldots\right)$.

\subsubsection{First system}

Here is the characteristic presentation of the first system.

$V_{x}^{1}=0, V_{y}^{1}=0, V_{u}^{1}=0, V_{x}^{2}=0, V_{y}^{2}=0, V_{u}^{2}=0, V^{3}=0, H_{x}=0, H_{y}=0$

There is no differential equation in $H$ alone (except the two ones we have introduced above). This case corresponds to the general case. The solutions of the $V$ 's are

$$
\begin{aligned}
& V^{1}(x, y, u)=C V^{1}, \\
& V^{2}(x, y, u)=C V^{2}, \\
& V^{3}(x, y, u)=0 .
\end{aligned}
$$

The allowed transformations are translations in the $(x, y)$ plane $(\lambda, \mu$ denote constants):

$$
X=x+\lambda, \quad Y=y+\mu, \quad U=u .
$$

\subsubsection{Second system}

Here is the characteristic presentation of the second system.

$$
\begin{aligned}
& V_{x}^{1}=-\frac{V^{3} H_{u}}{H}, V_{y}^{1}=0, V_{u}^{1}=0, V_{x}^{2}=0, V_{y}^{2}=-\frac{V^{3} H_{u}}{H}, V_{u}^{2}=0, V_{x}^{3}=0, V_{y}^{3}=0, \\
& V_{u}^{3}=-\frac{-V^{3} H_{u}{ }^{2}+H_{u u} H V^{3}}{H_{u} H}, H_{u u u}=-\frac{-2 H H_{u u}{ }^{2}+H_{u}{ }^{2} H_{u u}}{H_{u} H}, H_{x}=0, H_{y}=0 .
\end{aligned}
$$


This case corresponds to any function $H$ which satisfies the third order differential equation above. Computing Taylor expansions of solutions we get

$$
\begin{aligned}
V^{1}(x, y, u) & =C V^{1}-\frac{x C V^{3} C H_{u}}{C H}, \\
V^{2}(x, y, u) & =C V^{2}-\frac{y C V^{3} C H_{u}}{C H}, \\
V^{3}(x, y, u) & =C V^{3}-\frac{u\left(-C V^{3} C H_{u}{ }^{2}+C H_{u u} C H C V^{3}\right)}{C H_{u} C H}
\end{aligned}
$$

The Lie algebra has dimension three i.e. the solutions depend on the three arbitrary constants $C V^{1}, C V^{2}$ and $C V^{3}$ (the constants which appear in the Taylor expansion of $H$ are supposed to be known).

$V=C V^{1}\left(\begin{array}{l}1 \\ 0 \\ 0\end{array}\right)+C V^{2}\left(\begin{array}{l}0 \\ 1 \\ 0\end{array}\right)+C V^{3}\left(\begin{array}{c}-\frac{x C H_{u}}{C H} \\ -\frac{y C H_{u}}{C H} \\ C H_{u} C H+u\left(C H_{u}{ }^{2}-C H_{u u} C H\right)\end{array}\right)$

Remark we find again (setting $C V^{3}=0$ ) the Lie symmetries of section 8.1.1. Some other symmetries exist however in this particular case. A class of functions $H$ which satisfy the third order differential equation above is given by

$$
H(u)=\alpha u+\beta
$$

where $\alpha, \beta$ are constants (actually $\alpha=C H_{u}$ and $\beta=C H$ here since solutions have been expanded at the origin). Setting $C H_{u}=C H=1$ we find the symmetry group

$$
V=C V^{1}\left(\begin{array}{l}
1 \\
0 \\
0
\end{array}\right)+C V^{2}\left(\begin{array}{l}
0 \\
1 \\
0
\end{array}\right)+C V^{3}\left(\begin{array}{c}
-x \\
-y \\
u+1
\end{array}\right)
$$

The flows generated by the two first vector fields are translations in the $(x, y)$ plane. The third vector field generates the group of dilatations (where $\lambda$ denotes a constant)

$$
X=\frac{x}{\lambda}, \quad Y=\frac{y}{\lambda}, \quad U+1=\lambda(u+1) .
$$




\subsubsection{Third system}

The third regular differential system correspond to the case $H(u)=$ constant.

$$
\begin{gathered}
V_{x x}^{2}=0, V_{x x}^{3}=H V_{y}^{3}+V_{y y}^{3}, V_{x u}^{3}=-\frac{1}{2} V_{x}^{2} H, V_{y u}^{3}=0, V_{u u}^{3}=0, V_{x}^{1}=0, \\
V_{y}^{1}=V_{x}^{2}, V_{u}^{1}=0, V_{y}^{2}=0, V_{u}^{2}=0, H_{x}=0, H_{y}=0, H_{u}=0 .
\end{gathered}
$$

The solutions of the $V$ 's are

$$
\begin{aligned}
V^{1}(x, y, u)= & C V^{1}+y C V_{x}^{2}, \\
V^{2}(x, y, u)= & C V^{2}+x C V_{x}^{2}, \\
V^{3}(x, y, u)= & C V^{3}+x C V_{x}^{3}+y C V_{y}^{3}+u C V_{u}^{3}+\frac{1}{2} x^{2}\left(C H C V_{y}^{3}+C V_{y y}^{3}\right) \\
& +x y C V_{x y}^{3}-\frac{1}{2} x u C V_{x}^{2} C H+\frac{1}{2} y^{2} C V_{y y}^{3}+\cdots
\end{aligned}
$$

The vector fields associated to $C V^{1}$ and $C V^{2}$ generate the translations we already met in the general case. The vector field associated to $C V_{x}^{2}$ generates an hyperbolic rotation

$$
\left(\begin{array}{l}
X \\
Y
\end{array}\right)=\left(\begin{array}{ll}
a & b \\
b & a
\end{array}\right)\left(\begin{array}{l}
x \\
y
\end{array}\right), \quad U=u e^{-\frac{1}{2}(Y-y)}
$$

where $a^{2}-b^{2}=1$. The vector field associated to $C V_{u}^{3}$ generates the group of dilatations $U=\lambda u$. The other symmetries depend on an arbitrary solution $\alpha(x, y)$ of the equation $\mathrm{E}_{H}$ since it is linear in this case (see [Olver, 1993, page 124]):

$$
V=\alpha(x, y) \frac{\partial}{\partial u}
$$

\subsubsection{Fourth system}

The fourth system corresponds to the wave equation $(H(u)=0)$. There are still more symmetries than in the third case. See [Olver, 1993, page 124] for their descriptions.

$$
\begin{gathered}
V_{x x}^{2}=V_{y y}^{2}, V_{x x}^{3}=V_{y y}^{3}, V_{x u}^{3}=0, V_{y u}^{3}=0, V_{u u}^{3}=0, V_{x}^{1}=V_{y}^{2}, V_{y}^{1}=V_{x}^{2}, \\
V_{u}^{1}=0, V_{u}^{2}=0, H=0 .
\end{gathered}
$$

\section{Conclusion}

We have described an algorithm which computes a representation of the radical $\mathfrak{p}$ of any finitely generated differential ideal as an intersection of radical differential ideals. The representation separates the minimal differential 
prime components of $\mathfrak{p}$ which do not have the same dimension. It permits to compute Taylor expansions of solutions of $\mathfrak{p}$ and the Hilbert's polynomials associated to its minimal differential prime components. The algorithm is implemented in MAPLE within a package. Its implementation is quite tricky: it applies an analogue of Buchberger's second criterion, it manages to perform Gröbner bases computations in dimension zero and is able to reuse a representation of $\mathfrak{p}$ for a ranking to simplify the computation of a representation of $\mathfrak{p}$ for another ranking. Quite surprisingly, the algebraic computations turn out to be much easier to handle than one might fear. In order to prove and present our algorithm, we had to improve some of Kolchin's theorems. Our results (e.g. Lazard's Lemma) do not only apply in differential algebra but also for the non differential commutative algebra. Remark this phenomenon is not new: Ritt's characteristic sets theory, first developed for differential equations, has become later very popular for systems of usual polynomials.

\section{References}

Thomas Becker and Volker Weispfenning. Gröbner Bases: a computational approach to commutative algebra, volume 141 of Graduate Texts in Mathematics. Springer Verlag, 1991.

François Boulier. Étude et implantation de quelques algorithmes en algèbre différentielle. $\mathrm{PhD}$ thesis, Université Lille I, 59655, Villeneuve d'Ascq, France, 1994. http://tel.archives-ouvertes.fr/tel-00137866.

François Boulier. Some improvements of a lemma of Rosenfeld. (never published), 1997.

François Boulier, Daniel Lazard, François Ollivier, and Michel Petitot. Representation for the radical of a finitely generated differential ideal. In ISSAC'95: Proceedings of the 1995 international symposium on Symbolic and algebraic computation, pages 158-166, New York, NY, USA, 1995. ACM Press. ISBN 0-89791-699-9. http://hal .archives-ouvertes.fr/ hal-00138020.

Driss Bouziane, Abdelillah Kandri Rody, and Hamid Maârouf. UnmixedDimensional Decomposition of a Finitely Generated Perfect Differential Ideal. Journal of Symbolic Computation, 31:631-649, 2001.

Bruno Buchberger. A criterion for detecting unnecessary reductions in the construction of Gröbner bases, volume 72 of Lecture Notes in Computer Science, pages 3-21. Springer Verlag, 1979. 
Giuseppa Carra-Ferro. Gröbner bases and differential ideals. In Notes of $A A E C C$ 5, pages 129-140, Menorca, Spain, 1987. Springer Verlag.

David Cox, John Little, and Donal O'Shea. Ideals, Varieties and Algorithms. An introduction to computational algebraic geometry and commutative algebra. Undergraduate Texts in Mathematics. Springer Verlag, New York, 1992.

David Eisenbud. Commutative Algebra with a View Toward Algebraic Geometry, volume 150 of Graduate Texts in Mathematics. Springer Verlag, 1995.

Giovanni Gallo, Bubaneshwar Mishra, and François Ollivier. Some constructions in rings of differential polynomials, volume 539 of Lecture Notes in Computer Science, pages 171-182. , Montréal, Canada, 1991.

R. Gebauer and H. M. Möller. On an Installation of Buchberger's Algorithm. Journal of Symbolic Computation, 6(2\&3):275-286, October/December 1988.

Évelyne Hubert. Étude Algébrique et Algorithmique des Singularités des Équations Différentielles Implicites. PhD thesis, Institut National Polytechnique de Grenoble, France, 1997.

Maurice Janet. Systèmes d'équations aux dérivées partielles, volume 3 of Journal de Mathématiques, $8^{e}$ série. Gauthier-Villars, Paris, 1920.

Maurice Janet. Leçons sur les systèmes d'équations aux dérivées partielles, volume IV of Cahiers Scientifiques. Gauthier-Villars, Paris, 1929.

Mickael Kalkbrener. A Generalized Euclidean Algorithm for Computing Triangular Representations of Algebraic Varieties. Journal of Symbolic Computation, 15:143-167, 1993.

Donald Erwin Knuth. The art of computer programming. Addison-Wesley, 1966. Second edition.

Ellis Robert Kolchin. Differential Algebra and Algebraic Groups. Academic Press, New York, 1973.

D. König. Theorie der endlichen und unendlichen Graphen. Chelsea publ. Co., New York, 1950.

Daniel Lazard. Solving Zero-dimensional Algebraic Systems. Journal of Symbolic Computation, 13:117-131, 1992. 
Computing representations for radicals of finitely generated differential ideals 55

H. Levi. The low power theorem for partial differential equations. Annals of the Mathematical Society, 46:113-119, 1945.

Hamid Maârouf. Étude de Quelques Problèmes Effectifs en Algèbre Différentielle. PhD thesis, Université Cadi Ayyad, Morocco, 1996.

Elizabeth L. Mansfield. Differential Gröbner Bases. PhD thesis, University of Sydney, Australia, 1991.

Marc Moreno Maza. Calculs de Pgcd au-dessus des Tours d'Extensions Simples et Résolution des Systèmes d'Équations Algébriques. PhD thesis, Université Paris VI, France, 1997.

Sally Morrison. Yet another proof of Lazard's lemma. private communication, december 1995.

Sally Morrison. The Differential Ideal $[P]: M^{\infty}$. Journal of Symbolic Computation, 28:631-656, 1999.

François Ollivier. Le problème de l'identifiabilité structurelle globale : approche théorique, méthodes effectives et bornes de complexité. $\mathrm{PhD}$ thesis, École Polytechnique, Palaiseau, France, 1990.

François Ollivier. A proof of Lazard's lemma. private communication, october 1998.

Peter J. Olver. Applications of Lie groups to differential equations, volume 107 of Graduate Texts in Mathematics. Springer Verlag, second edition, 1993.

Peter J. Olver. Equivalence, Invariants and Symmetry. Cambridge University Press, New York, 1995.

Ariane Péladan-Germa. Tests effectifs de Nullité dans des extensions d'anneaux différentiels. $\mathrm{PhD}$ thesis, École Polytechnique, Palaiseau, France, 1997.

Gregory J. Reid. Algorithms for reducing a system of PDEs to standard form determining the dimension of its solution space and calculating its Taylor series solution. Eur. J. of Applied Math., 2:293-318, 1991.

Gregory J. Reid, Allan D. Wittkopf, and Alan Boulton. Reduction of systems of nonlinear partial differential equations to simplified involutive forms. European Journal of Applied Math., pages 604-635, 1996. 
Gregory J. Reid, Ping Lin, and Allan D. Wittkopf. Differential EliminationCompletion Algorithms for DAE and PDAE. Studies in Applied Mathematics, 106(1):1-45, 2001.

Charles Riquier. Les systèmes d'équations aux dérivées partielles. GauthierVillars, Paris, 1910.

Joseph Fels Ritt. Differential equations from the algebraic standpoint, volume 14 of American Mathematical Society Colloquium Publications. AMS, New York, 1932.

Joseph Fels Ritt. Differential Algebra. Dover Publications Inc., New York, 1950. http://www.ams.org/online_bks/coll33.

Azriel Rosenfeld. Specializations in differential algebra. Trans. Amer. Math. Soc., 90:394-407, 1959.

Josef Schicho and Ziming Li. A construction of radical ideals in polynomial algebra. Technical report, RISC, Johannes Kepler University, Linz, Austria, august 1995.

Abraham Seidenberg. Some basic theorems in differential algebra (characteristic $p$ arbitrary). Trans. Amer. Math. Soc., 73:174-190, 1952.

Abraham Seidenberg. An elimination theory for differential algebra. Univ. California Publ. Math. (New Series), 3:31-65, 1956.

Abraham Seidenberg. Abstract differential algebra and the analytic case. Proc. Amer. Math. Soc., 9:159-164, 1958.

Abraham Seidenberg. Abstract differential algebra and the analytic case II. Proc. Amer. Math. Soc., 23:689-691, 1969.

Bruno Louis van der Waerden. Algebra. Springer Verlag, Berlin, seventh edition, 1966.

Dongming Wang. An elimination method for differential polynomial systems I. Technical report, LIFIA-IMAG, Grenoble, France, 1994.

Wen Tsün Wu. On the foundation of algebraic differential geometry. Mechanization of Mathematics, research preprints, 3:2-27, 1989.

Oscar Zariski and Pierre Samuel. Commutative Algebra. Van Nostrand, New York, 1958. Also volumes 28 and 29 of the Graduate Texts in Mathematics, Springer Verlag. 


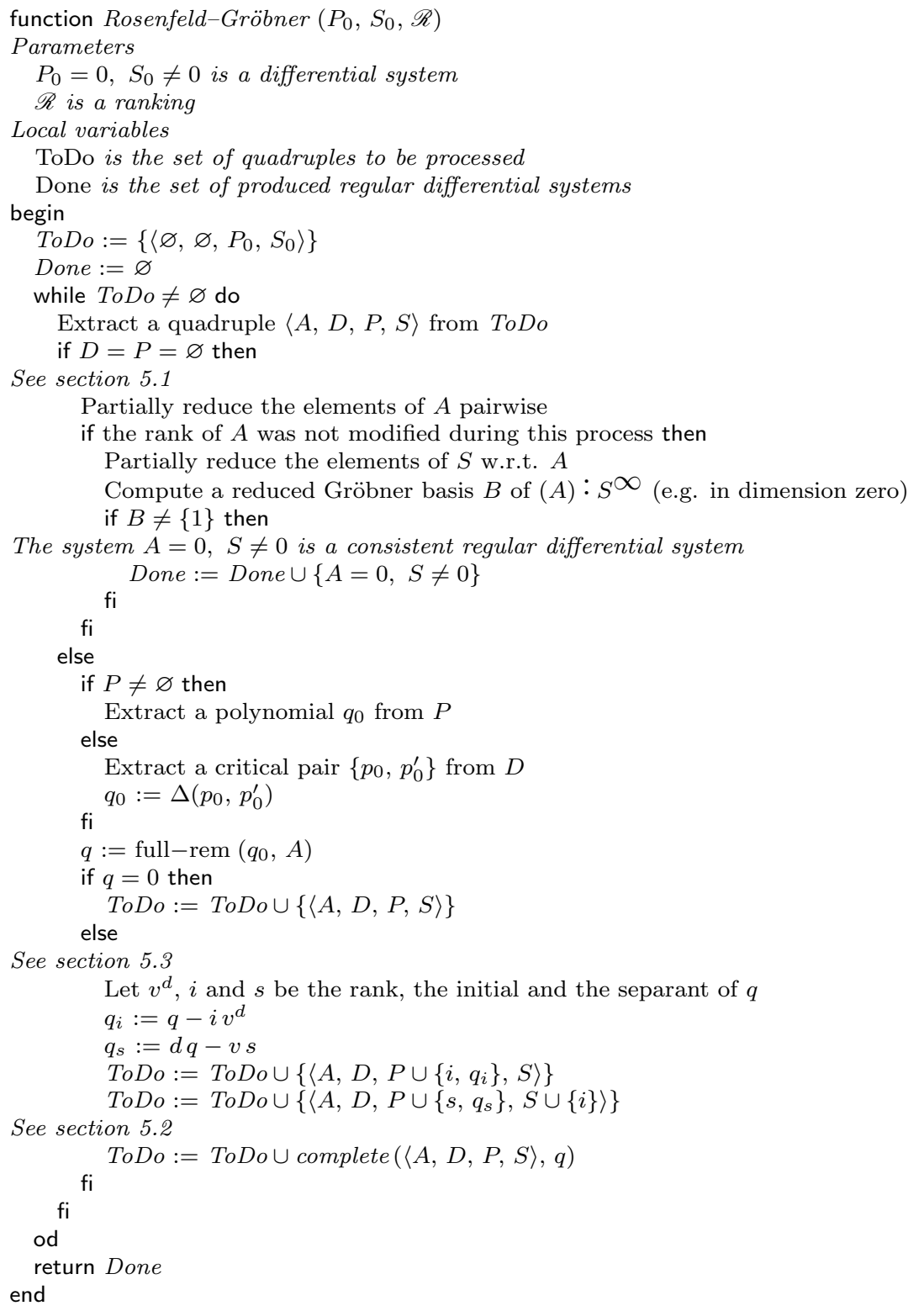


function complete $\left(\left\langle A, D^{*}, P^{*}, S\right\rangle, q\right)$

Parameters

$\left\langle A, D^{*}, P^{*}, S\right\rangle$ is a quadruple (see Section 5.2)

$q$ is a nonzero differential polynomial reduced w.r.t. $A$

begin

$A^{\prime}:=\{q\} \cup\{p \in A \mid \operatorname{ld} p$ is not a derivative of $\operatorname{ld} q\}$.

$D_{0}:=$ the set of all the critical pairs that can be formed with $q$ and any $p \in A$

$D_{1}$ is any subset of $D_{0}$ that can be obtained as follows:

a critical pair $\{p, q\} \in D_{0}$ is not kept in $D_{1}$ only if condition (a) or (b) holds:

(a) $p$ and $q$ are linear homogeneous differential polynomials

in one differential indeterminate with constant coeffi-

cients such that $\operatorname{lcm}(\theta, \phi)=\theta \phi$ (where $\operatorname{ld} q=\theta u$ and $\operatorname{ld} p=\phi u)$,

(b) there exists a critical pair $\left\{p^{\prime}, q\right\} \in D_{1}$ such that the triple $\left\langle q, p^{\prime}, p\right\rangle$ satisfies the hypotheses $\mathbf{H 1}, \mathbf{H} \mathbf{2}$ and $\mathbf{H 3}$ of Proposition 27.

$D_{2}$ is any subset of $D^{*}$ that can be obtained as follows:

a critical pair $\left\{p, p^{\prime}\right\} \in D^{*}$ is not kept in $D_{2}$ only if the triple $\left\langle p, q, p^{\prime}\right\rangle$ satisfies the hypotheses $\mathbf{H 1}, \mathbf{H 2}$ and $\mathbf{H 3}$ of Proposition 27 and $\operatorname{lcd}\left(\operatorname{ld} p, \operatorname{ld} p^{\prime}\right)$

is different from both $\operatorname{lcd}(\operatorname{ld} p, \operatorname{ld} q)$ and $\operatorname{lcd}\left(\operatorname{ld} p^{\prime}, \operatorname{ld} q\right)$.

$A$ detailed implementation is given in Section 5.5

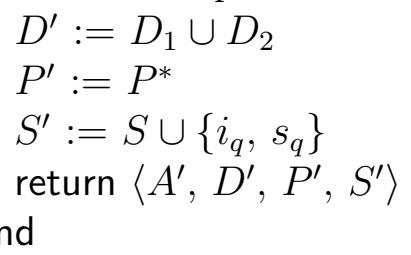

Figure 2: The complete function 
$D_{1}:=\emptyset$

while $D_{0} \neq \varnothing$ do

pick a critical pair $\{p, q\} \in D_{0}$

$D_{0}:=D_{0} \backslash\{\{p, q\}\}$

if $p, q$ are linear homogeneous differential polynomials in one differential indeterminate and with constant coefficients or if there does not exist any critical pair $\left\{p^{\prime}, q\right\} \in D_{0} \cup D_{1}$ such that the triple $\left\langle q, p^{\prime}, p\right\rangle$ satisfies the hypotheses $\mathbf{H 1}$ to $\mathbf{H} 3$ of Proposition 27 then $D_{1}:=D_{1} \cup\{\{p, q\}\}$

fi

od

Remove from $D_{1}$ all pairs $\{p, q\}$ such that $p, q$ are linear homogeneous differential polynomials in one differential indeterminate and with constant coefficients

Let $D_{2}$ be the subset of all the critical pairs $\left\{p, p^{\prime}\right\} \in D^{*}$ such that $\left\langle p, q, p^{\prime}\right\rangle$ does not satisfy the hypotheses H1, H2 and H3 of Proposition 27 or such that $\operatorname{lcd}\left(\operatorname{ld} p, \operatorname{ld} p^{\prime}\right)$ is equal to $\operatorname{lcd}(\operatorname{ld} p, \operatorname{ld} q)$ or to $\operatorname{lcd}\left(\operatorname{ld} p^{\prime}, \operatorname{ld} q\right)$.

$D^{\prime}:=D_{1} \cup D_{2}$.

Figure 3: Implementation details for function complete. 